\title{
Beyle-Stendhal en 1809 : les énigmes de la Campagne de Vienne
}

\section{Elaine Williamson}

\section{(2) OpenEdition}

12 Journals

Édition électronique

URL : http://journals.openedition.org/recherchestravaux/473

DOI : 10.4000/recherchestravaux.473

ISSN : 1969-6434

Éditeur

UGA Éditions/Université Grenoble Alpes

\section{Édition imprimée}

Date de publication : 15 décembre 2011

Pagination : 35-71

ISBN : 978-2-84310-215-8

ISSN : 0151-1874

\section{Référence électronique}

Elaine Williamson, «Beyle-Stendhal en 1809 : les énigmes de la Campagne de Vienne », Recherches \& Travaux [En ligne], 79 | 2011, mis en ligne le 15 juin 2013, consulté le 08 septembre 2020. URL : http:// journals.openedition.org/recherchestravaux/473; DOI : https://doi.org/10.4000/recherchestravaux. 473 
Elaine Williamson

London Institute in Paris

\section{Beyle-Stendhal en I809 : les énigmes de la Campagne de Vienne}

À partir de quels documents construisons-nous notre idée, notre vision des activités de Beyle-Stendhal au cours de l'année 1809, et tout particulièrement pendant la campagne d'Autriche? Nos sources principales sont constituées par son journal et par sa correspondance, textes qui se complètent et se relaient de telle sorte qu'ils deviennent parfois presque interchangeables : des pages du journal seront envoyées en guise de lettre à sa sœur Pauline ou à son ami Félix Faure et, inversement, certaines des lettres qu'il écrit ou qu'il reçoit seront recopiées dans le journal. Cette pratique transgénérique, Beyle le constate lui-même, ne va pourtant pas sans inconvénients. Telle lettre destinée à être reprise dans son journal «manque, dira-t-il, de profondeur et est enjolivé[e $]^{\mathrm{T}}$ ». Le contenu de telle autre est jugé "vrai et au style près bon pour mon journal ${ }^{2}$ », la restriction concernant le style étant apportée en raison de l'absence des "détails mathématiques et sévères", constituants essentiels de son écriture diaristique, qui devaient lui permettre de présenter de façon objective «le Procès-verbal de [sa] manière d'être ${ }^{3}$ ».

La transgénéricité fausse donc parfois sa pensée, elle cache ou efface certains éléments importants de l'histoire de sa vie et de sa personnalité. Certes, le courrier que lui avaient adressé sa famille et ses amis, ainsi que le journal rédigé par Faure pendant son séjour à Vienne ${ }^{4}$, où il avait rejoint Beyle au

I. Journal, 5 mai I809. BMG, R. 5896, vol. 5, fo $103 v^{\circ}$; OI, t. I, p. 532. Nous respectons l'orthographe et la ponctuation des manuscrits autographes.

2. Journal, début juin I8Io. BMG, R. 9982, vol. 3, fo IO220 ; OI, t. I, p. 589.

3. Ibid., p. 590.

4. OI, t. I, p. I049-I057. 
mois d'août, nous apportent des compléments d'information précieux. Il reste néanmoins beaucoup de zones d'ombre, d'incertitude : des cahiers du journal écrits entre le 15 mai et le 20 octobre 1809 ont été perdus, des lettres aussi sans doute. Parmi les documents conservés, la lettre faisant fonction de journal, on l'a vu, ne sera pas toujours assez profonde, assez vraie, assez mathématiquement précise; quant au texte du journal proprement dit, normalement censé être réservé à son usage personnel, il sera souvent elliptique, voire mystificateur. Beyle-Stendhal, on le sait, pratique le non-dit - un nondit parfois ouvertement déclaré, un refus de dire clairement exprimé. Les omissions volontaires et, dans son journal, les espaces blancs plus ou moins grands réservés à des développements ultérieurs, laissent donc persister des doutes, créent des énigmes. Il en va de même des phrases ambiguës, des alibis destinés à fourvoyer le lecteur indiscret, de toute la panoplie de jeux rhétoriques qu'il met en ouvre et que lui dictent non seulement un esprit ludique mais aussi et surtout une prudence infinie, invétérée.

Afin de tenter de résoudre quelques-unes des énigmes ainsi créées à propos de l'année I809, nous avons effectué un dépouillement systématique de plusieurs séries de manuscrits conservées dans des archives publiques ou privées en France et en Autriche, notamment à Paris, aux Archives nationales, aux Archives du ministère des Affaires étrangères et à la Bibliothèque Thiers; à Vincennes, au service historique de l'armée de terre; et à Vienne, à l'Österreichisches Staatsarchiv. Il s'agissait surtout de déterminer la nature précise des fonctions remplies par Beyle pendant la campagne d'Autriche. Nos recherches ont permis d'ajouter plusieurs manuscrits autographes inédits au corpus que nous avions déjà pu réunir et qui fait l'objet d'une édition critique actuellement en préparation. Elles ont également éclairé d'un jour nouveau certains aspects peu connus de la vie de Beyle à Vienne.

Notre propos dans le présent article est de centrer notre interrogation sur trois questions principales :

I. Beyle a-t-il réellement travaillé avec Napoléon, comme il le prétend à plusieurs reprises dans son journal?

2. A-t-il été chargé, en juillet I809, d'une mission dont le but était d'offrir la couronne de Hongrie à un prince hongrois ou à un archiduc autrichien, comme il l'affirme dans sa correspondance des années I830?

3. A-t-il pris part aux négociations du mariage de Napoléon et de l'archiduchesse Marie-Louise, comme nous le dit Romain Colomb?

Ces questions ne relèvent pas exclusivement de la biographie. Comme on le verra, ce sont les processus d'écriture, la représentation non seulement de soi mais également de l'histoire, l'articulation du vrai, du faux, et du vrai- 
faux, qui entrent en jeu ici, éléments essentiels à la compréhension du Beyle qui allait devenir Stendhal.

Suivons-le donc dans la campagne de i809.

Elle commencera pour Beyle vers la fin du mois de mars. Les semaines précédentes, il avait été employé à Paris comme secrétaire-rédacteur dans les bureaux de l'intendance de la Maison de l'empereur, où l'intendant général, son cousin Pierre Daru, l'avait fait participer à des affaires aussi diverses que la commande de tableaux destinés à la galerie de Diane au palais des Tuileries', ou le travail préparatoire - terme dont on verra l'importance par la suite - à la composition de la liste civile en Toscane ${ }^{6}$ (fig. I, p. 57-58).

Le 24 mars, le comte Daru sera nommé intendant général de l'armée d'Allemagne. Le 27 - c'est le jour où l'archiduc Charles appelle les peuples allemands à l'insurrection, mais la guerre n'est pas officiellement déclarée -, Beyle s'apprête à quitter Paris. Il annonce son départ imminent à sa sœur ${ }^{7}$ et commence à s'occuper des affaires autrichiennes : c'est lui qui datera de sa main une lettre autographe de Daru à Champagny, alors ministre des Relations extérieures, au sujet des contributions et réquisitions à prélever sur les terres possédées en Allemagne par des seigneurs attachés à la maison d'Autriche ${ }^{8}$.

Le lendemain, 28 mars, Daru reçoit l'ordre de se rendre à Strasbourg, où le suivront les commissaires des guerres affectés à son service. Beyle, qui a le grade d'adjoint aux commissaires des guerres, sera du nombre et y arrivera le 2 ou le 3 avril. Le 5, à deux heures du matin, il écrit à Faure sur un ton plutôt léger : "Je me promène depuis minuit en long et en diagonale dans un salon sans feu : je gèle, mais j'ai l'avantage d'être en grande tenue.» Il grelotte, la demie sonne mais, annonce-t-il, «je reste à mon poste ${ }^{9}$. Et son travail de secrétaire-rédacteur auprès de Daru se poursuit, sans doute jusqu'à une heure encore plus avancée - si l'empereur lui-même écrivait des lettres à toute heure de la nuit ${ }^{\mathrm{ro}}$, Daru et ses collaborateurs se devaient d'être aussi actifs.

5. Voir E. Williamson, «Stendhal et Dominique Vivant Denon. De l'expédition d'Égypte à l'inventaire du musée Napoléon. Lettres et documents inédits ", Stendhal Club, n ${ }^{\circ} 23$, I5 avril I989, p. I72-I74.

6. Nous reproduisons plus loin en fac-similé un des projets de rapport rédigés par Beyle dans le cadre de ce travail préparatoire; pour la transcription de tous les projets autographes de rapport et de décret sur la Toscane, voir E. Williamson, "Stendhal et l'Italie napoléonienne : rêves et réalités (d'après des documents autographes inédits) ", dans Stendhal, Paris et le mirage italien, Bibliothèque historique de la Ville de Paris, I992, p. 235-257.

7. CG, t. I, p. 818-819; fac-similé de la lettre publié par N. Gotteri dans Claude Petiet, ministre de la Guerre, intendant général de la Grande Armée et ses fils Alexandre, Auguste et Sylvain, SPM, coll. «Kronos», I999, annexe III.

8. AMAE, Correspondance politique, Autriche, vol. $384, f^{\circ} 52$.

9. $C G$, t. I, p. 819-820.

IO. L'heure est souvent indiquée à la suite de la date dans les lettres écrites en campagne; 
Ce 5 avril, donc, Beyle écrira de sa main, mais au nom de l'intendant général, une lettre en grande partie inédite qui sera signée par Daru et adressée au maréchal Berthier, prince de Neuchâtel, futur prince de Wagram, vice-connétable et major-général de l'armée ${ }^{\mathrm{II}}$ (fig. 2, p. 59). À cette missive concernant l'organisation des subsistances de l'armée était joint un projet d'ordre du jour qui en fut séparé par la suite, mais que nous avons retrouvé sous une autre cote : il est également de la main de Beyle et, à notre connaissance, inédit. Berthier l'approuva en le paraphant « $A$ » et en le signant de son prénom "Alexandre", après y avoir apporté quelques ajouts ou corrections de sa main, y compris la transformation rapide, au moyen de deux traits, du «Projet d'Ordre du jour» en «Ordre» tout court ${ }^{12}$ (fig. 3, p. 60).

La question se pose, évidemment, de savoir si Beyle a écrit ces documents sous la dictée de Daru, à partir de notes fournies par celui-ci, ou s'il en a composé lui-même le texte. Pour essayer de répondre à cette question, il faut en poser une autre : qui est cet Henri Beyle qui suit le comte Daru à Strasbourg? Un simple adjoint aux commissaires des guerres à qui il ne faut confier aucune responsabilité importante? C'est ainsi qu'on le représente quelquefois, en partie sans doute parce qu'il ne répugne pas à divulguer dans son journal ou sa correspondance les étourderies dont il se rend parfois coupable et qui le privent pendant quelque temps de la bienveillance de Daru. Mais à s'en tenir à cette représentation réductrice de son comportement, on oublie qu'il avait déjà fait ses preuves comme agent de l'administration impériale en dirigeant, en I808, celle des domaines situés dans le département de l'Ocker. Ses capacités de rédacteur avaient été reconnues à l'époque, et l'on savait donc, en I809, qu'on pouvait le charger de préparer des projets de lettres, de rapports et d'ordres du jour destinés à être signés par ses supérieurs. C'est là, d'ailleurs, il convient de le souligner, une méthode de travail attestée à tous les niveaux de l'administration napoléonienne. Ainsi le maréchal Berthier, comme tous les ministres, y compris Daru, soumettait communément à la signature de Napoléon des projets écrits au nom de l'empereur. Et Daru, comme tous ses collègues, demandait fréquemment à ses secrétaires de lui rédiger des documents destinés à recevoir sa signature.

Quelle est la méthode de travail adoptée par Daru au commencement de la campagne d'Autriche? La dictée? C'est peu probable. L'intendant général

on relève ainsi, pour le début de la campagne d'Autriche en avril ı809, douze lettres rédigées pendant la nuit et au petit matin (Voir CN, t. XVIII, p. 473-516).

II. ASHAT, 2 C 87. Cette lettre a été partiellement reproduite d'après une copie, et sans allusion à Stendhal, par le commandant Charles Saski dans Campagne de I809 en Allemagne et en Autriche, t. II (I900), Paris-Nancy, Berger-Levrault et Cie, I899-I902, p. 50-5I, note 2.

I2. ASHAT, I7 C 82. 
avait alors une somme de travail exceptionnelle à fournir dans des délais très courts. A-t-il donc communiqué à Beyle des instructions écrites sous forme de notes? C'est possible - on connaît quelques billets de ce genre adressés nommément à Beyle par Daru en I8Io et $\mathrm{I} 8 \mathrm{II}^{\mathrm{I3}}$ (fig. 4, p. 6I), des apostilles inscrites par ce dernier sur des rapports signés «De Beyle ${ }^{14}$ » (fig. 5, p. 62), ainsi que des instructions plus élaborées ${ }^{15}$ (fig. 6, p. 63). Ou bien, Beyle est-il lui-même l'auteur de ces textes? Ce n'est pas impossible. Déjà à Brunswick, il avait écrit de nombreuses lettres transmettant des renseignements, voire des instructions, à des ministres, des généraux, des colonels, des préfets ${ }^{16}$. Il convient de rappeler dans ce contexte que certains des rapports et projets de décrets soumis à Napoléon par Daru en I8Io et I8II auront été composés par Beyle ${ }^{17}$ et que ce dernier avait déjà contribué, au mois de mars I809, à la préparation des décrets portant sur la liste civile en Toscane ${ }^{\mathrm{r} 8}$.

Il est rarement aisé d'identifier avec certitude l'auteur des documents administratifs ainsi rédigés. En témoigne un long rapport à Berthier en date du 9 avril I809, qui est entièrement de la main de Beyle mais qui porte la signature de Daru ${ }^{19}$ (fig. 7, p. 64). Inconnu, semble-t-il, des exégètes stendhaliens, ce texte fut publié en 1900 par le commandant Saski mais sous une autre forme et avec quelques omission ${ }^{20}$. Le rapport présenté par Saski est en effet adressé à l'empereur et serait conservé aux Archives nationales. Comme nous n'y avons pas retrouvé l'original, nous ignorons s'il était également de la main de Beyle; on est donc tenté de supposer que sa tâche avait consisté simplement à le recopier en y apportant les modifications nécessitées par le changement de destinataire. Si la version qu'il a écrite comporte quelques inadvertances assez lourdes de conséquences (chiffrer, par exemple, en millions au lieu de

I3. AN, $\mathrm{O}^{2} \mathrm{IO}_{2}, \mathrm{n}^{\circ}$ 9, 74, 75, 73, Daru à Beyle, notes griffonnées sur des feuilles volantes, s. d. [2I-24 novembre I8Io, début et mi-janvier I8II]. Voir E. Williamson, Stendhal et la Hollande : Correspondance administrative inédite, I8I0-1812, Londres, Institute of Romance Studies, 1996, p. 247, 255, 284.

I4. Ibid., $\mathrm{n}^{\mathrm{o}}$ I04, rapport de Beyle s. d., apostille marginale de Daru [I3 mai I8II] ; ibid., $\mathrm{n}^{\circ}$ 97, rapport de Beyle s. d. [6-7 juin I8II], apostilles marginales de Daru et de Beyle; voir

E. Williamson, ouvr. cité (note I3 supra), p. I29, I36-I37.

I5. Ibid., n ${ }^{\circ}$ I07, note autographe de Daru à Beyle, s. d. [2 avril I8II]; voir E. Williamson, ouvr. cité (note I3 supra), p. I09-IIo.

I6. BMG, R. 296 vol. I et 2 ; $C G$, t. I, p. 64I-80o.

17. Voir, par exemple, E. Williamson, ouvr. cité (note I3 supra), p. 45-46, 97-99, I30-133, 201 .

I8. Voir E. Williamson, ouvr. cité (note 6 supra).

19. ASHAT 2 C 87. Les première, avant-dernière et dernière pages de ce rapport sont reproduites plus loin (fig. 7, p. 64).

20. Ouvr. cité (note II supra), p. 76-84. 
centaines de milliers le nombre de rations de biscuit en réserve à Augsbourg), la version publiée par le commandant Saski n'en est pas exempte (confusion entre la France et la Franconie...), et l'on relève souvent des fautes de ce genre (attribuables sans doute à l'indispensable rapidité de la rédaction) dans les documents écrits en campagne par d'autres secrétaires, voire des ministres. Il n'est donc pas impossible que Beyle soit l'auteur du texte originel. On y remarque, notamment, un style qui rappelle en plusieurs points celui de Beyle-Stendhal. Ainsi, dans le premier paragraphe, il expose d'une manière claire le plan du rapport, conçu, précise-t-il, «pour faciliter la comparaison de ses résultats avec les ordres que S. M. a donnés». Daru, au contraire, préfère en général entrer plus rapidement dans le vif du sujet.

Quoi qu'il en soit, on constate qu'en ce début de campagne, Beyle remplit déjà une de ses fonctions constantes pendant l'année i809 : celle de secrétaire. C'est un aspect de son travail qu'il abordera relativement peu dans son journal ou dans sa correspondance personnelle, pour des raisons qui tiennent en partie à la politique et à une sorte de devoir de réserve qu'il s'impose à luimême. Le premier cahier de son journal de campagne qui nous soit parvenu se place en effet sous le signe de la discrétion. À la première page, on lit la note marginale suivante, inscrite perpendiculairement au texte :

\section{Parenthèse (Par prudence, ne sachant où mettre mes Papiers, Rien de politique, tous les noms changés $)^{21}$}

Le fac-similé de cette page, reproduite plus loin (fig. 8, p. 65), fait apparaitre des éléments codicologiques significatifs : l'encre et la plume utilisées pour rédiger la note marginale ainsi que le titre du journal sont différentes de celles utilisées dans le corps du texte - l'encre est plus noire, la plume un peu moins fine. Il en est de même, à tout le moins pour ce qui concerne l'encre, de plusieurs autres annotations consignées sur ce cahier. Le premier exemple que nous citerons est précieux car il est daté, de I8ı3 (fig. 9, p. 66) :

je travaillai sans cesse

avec L.emp. toutes mes

relations avec le grand homme

passées sous silence, pour ne pas me

compromettre.

$\underline{\mathrm{I} 8 \mathrm{I}^{22}}=$

2I. BMG, R. 5896, vol. 5, fo $116 v^{0} ; O I$, t. I, p. 517.

22. BMG, R. 5896, vol. 5 , fo IOgv $^{\circ}$; OI, t. I, p. 525 . 
Beyle-Stendhal en i809: les Énigmes de la Campagne de Vienne

Un deuxième exemple figure sur la couverture du cahier (fig. Io, p. 67) :

\section{Campagne}

de

Vienne en I809.

Par prudence je n'écrivais rien

$$
\begin{aligned}
& \text { I }^{\circ} \text { sur les év[énement]s militaires } \\
& 2^{\circ} \text { sur les relations politiques } \\
& \text { avec l'Allemagne } \\
& \text { et surtout la Prusse } \\
& \text { assez bête pour ne } \\
& \text { pas attaquer } \\
& 3^{\circ} \text { Les relations de } \\
& \text { Dom[inique] avec le plus } \\
& \text { grand des hommes. }
\end{aligned}
$$

Ceci n'est qu'un journal

destiné à m’observer moi-même,

nullement intéressant pour

d'autres ${ }^{23}$.

Notre troisième exemple est inscrit sur la quatrième de couverture (fig. II, p. 68) :

Campagne de 1809

de Strasbourg à Vienne.

Par Prudence rien de Politique, tous les noms changés, je ne notais que les ob. ons upon myself ${ }^{24}$.

Ces trois derniers exemples, on le voit, expriment la même idée principale que l'annotation marginale de la première page, à savoir la nécessité de la prudence. Mais seule l'annotation de la première page porte le signe et aussi la mention du mot «Parenthèse» (fig. 8, p. 65).

Or, dans l'édition de la Pléiade, la mise en page du texte et la référence donnée en note ${ }^{25}$ laissent à penser que cette annotation avait été consignée sur la couverture, à la suite du titre "Campagne de Vienne en I809" et des commentaires que Beyle y avait inscrits (fig. IO, p. 67). Petit détail sans grande importance? Pas tout à fait, à cause, justement, de la parenthèse. Car, comme

23. BMG, R. 5896, vol. 5, fo $117 \mathrm{v}^{\circ}$; OI, t. I, p. $5 \mathrm{I} 7$.

24. BMG, R. 5896, vol. 5, fo $96 \mathrm{r}^{\circ}$; $O I$, t. I, p. 539.

25. OI, t. I, p. 5 I7 et p. 1337 notes $c, 8$. 
l'a démontré François Michel ${ }^{26}$, et comme le fait remarquer Victor Del Litto à ce $\operatorname{propos}^{27}$, ce signe est souvent employé par Beyle pour désigner un alibi (au sens de "ruse» pour "faire diversion»), un code, donc, voire un mensonge. Si la parenthèse s'applique ici aux commentaires qui la précèdent, elle pourrait signifier que tout ce que dit Beyle sur la "prudence» et l'empereur, «le plus grand des hommes», est à traiter avec la plus grande précaution.

L'affaire se complique davantage parce qu'une autre note marginale, contemporaine du texte du journal, porte également une parenthèse ajoutée, après coup, à propos de l'empereur (fig. I2, p. 69) :

écrit à Ingolstadt (en revenant de chez l'empr.)

le 20 Avril à $9^{\text {h2 } 2}$.

On peut se demander par conséquent s'il ne s'agit pas, là aussi, d'une parenthèse introduite, en I813, pour marquer un alibi.

Que penser donc de ces déclarations de Beyle concernant ses relations avec l'empereur? Faut-il y voir, comme des exégètes du siècle dernier, une simple forfanterie, une affabulation, un alibi, un souvenir imprécis?

L'étude de l'itinéraire de l'empereur, ainsi que l'analyse des lettres et rapports conservés aux archives, permettent de constater qu'il n'est point impossible que Beyle ait en effet travaillé avec Napoléon en avril I809. Prenons le cas de son retour de chez l'empereur, le 20 du mois. Les biographes de Stendhal ont signalé qu'il n'aurait pu travailler avec Napoléon à Ingolstadt, le 20 avril, parce que ce dernier en était parti le 19. C'est exact. Mais l'empereur avait passé la nuit du 19 au 20 au château de Vohburg ${ }^{29}$, qui n’en était qu'à quinze kilomètres. Il est reparti, il est vrai, le 20, à sept heures et demie, mais il s'était levé à cinq heures, avait vu Berthier et écrit deux lettres datées de "Vohburg, 20 avril 1809 , six heures et demie du matin ${ }^{30} »$. Beyle aurait très bien pu s'y rendre à cheval le soir du I9 pour y travailler avec Napoléon et en repartir de bonne heure le lendemain. Notons, d'ailleurs, qu'il ne précise pas lui-même que l'empereur se trouvait encore à Ingolstadt le 20 avril.

Le récit des deux derniers jours que Beyle avait passés à Ingolstadt, les 20 et 2I avril, est repris dans son journal, le 23, à Landshut. C'est en marge de cette entrée qu'il écrira en $\mathrm{I} 8 \mathrm{I} 3$ « je travaillai sans cesse avec L.emp ${ }^{3}$ (fig. 9, p. 66).

26. Fr. Michel, «Les alibis de Stendhal», dans Etudes stendhaliennes [1958], $2^{\mathrm{e}}$ édition augmentée et présentée par V. Del Litto, Mercure de France, 1972, p. 32-47.

27. OI, t. I, p. I338, note Io p. 517 .

28. BMG, R. 5896, vol. 5, fo IIIV $^{0}$; OI, t. I, p. 523.

29. J. Tulard et L. Garros, Itinéraire de Napoléon au jour le jour, I769-I82I, Tallandier, I992, p. 3 I2.

30. CN, t. XVIII, p. 490, lettres au général Vandamme et au maréchal Masséna.

3I. Voir supra, p. 40. 
Mais de quel travail aurait-il été chargé, et pour quelle raison? Les mémoires du général Savary nous fournissent un indice. C'est également à Landshut, selon le général, que Napoléon se plaignait de l'absence de ses secrétaires :

L'empereur passa la journée à Landshut, ne faisant qu’y questionner tout le monde. Il s'impatientait de ne pas voir arriver ses secrétaires, ni le matériel de son cabinet. Il était venu depuis Paris avec une telle rapidité que rien n’avait pu le suivre ${ }^{32}$.

Dans ces circonstances, à Ingolstadt comme à Landshut, Beyle a pu remplacer un des secrétaires de l'empereur, ce qui expliquerait, autrement que par de la simple fanfaronnerie, les paroles par lesquelles il termine une lettre adressée à Félix Faure, le 26 avril, de Landshut : "Adieu, je cours voir S[a] M[ajesté] ${ }^{33}$." C'est le 26 avril, en effet, à 17 heures, que Napoléon revint à Landshut après avoir passé la journée à visiter les blessés à Egglofsheim ${ }^{34}$ et Eckmühl35.

Le lendemain, l'empereur et l'armée reprirent la marche sur l'Autriche. Pendant cette marche, Beyle remplit quelques missions auprès de Berthier et continua son travail de secrétaire-rédacteur. Puis, c'est l'arrivée et l'installation à Vienne, où il commence à travailler pour Martial Daru à la demande de celui-ci :

L'adorable Martial, écrit-il à Faure le I8 mai, [...] a été nommé intendant [de Vienne] avant-hier; ce matin il m’a demandé à son frère, comme étant au fait de sa manière de travailler. M. Daru a répondu : Fais la lettre, je la signerai ${ }^{36}$.

Notons au passage ce témoignage sur la manière dont le comte Pierre Daru faisait faire sa correspondance.

Beyle reprend auprès de Martial ses fonctions de secrétaire-rédacteur, tout en étant chargé de missions précises - dans le cadre, par exemple, des dispositions prises pour la surveillance du commerce visant à prévenir la contrebande et à protéger les subsistances, grains et fourrages contre le vol. Mais il n'en continue pas moins de travailler, à l'occasion, pour Pierre Daru, assurant, par exemple, la transcription de lettres dans le registre de la correspondance active de l'intendant général.

Le 3 juin, il se trouva associé par Daru à une opération d'une tout autre envergure : il s'agissait de l'opération de surveillance dont était l'objet le prince Kourakin, ambassadeur de Russie à Paris. L'affaire était d'importance, car une des préoccupations principales de l'empereur pendant la campagne d'Autriche

32. A.-J.-M.-R. Savary, Mémoires $d u d u c$ de Rovigo, pour servir à l histoire de Pempereur Napoléon, t. IV, A. Bossange, I828, p. 83-84.

33. $C G$, t. I, p. 823 .

34. Aujourd'hui Alteglofsheim.

35. J. Tulard et L. Garros, ouvr. cité (note 29 supra), p. 314.

36. $C G$, t. I, p. 833 . 
était la question de savoir s'il pouvait compter sur la Russie. Officiellement son allié depuis le traité de Tilsitt, le tsar ne lui avait pourtant pas apporté l'appui souhaité en Pologne, et Napoléon le soupçonnait de vouloir soutenir l'Autriche. Les dépêches de l'ambassadeur de France à Vienne, le général Andréossy, n'étaient pas faites pour calmer ces doutes. Dès le Is février I809, le général avait écrit : "Il est toujours beaucoup question de la Russie : la conduite de la plupart des Russes donnerait à penser que cette puissance n'est plus dans les mêmes principes ${ }^{37}$ ». Quelques mois plus tard, le 2 juin - c'était la veille du jour où Beyle devait prendre la plume à ce propos -, Champagny écrivait à Caulaincourt, ambassadeur de France à Saint-Pétersbourg : «L'Empereur ne veut pas que je vous cache que les dernières circonstances lui ont fait beaucoup perdre de la confiance que lui inspirait l'alliance de la Russie, et qu'elles sont pour lui des indices de la mauvaise foi de ce Cabinet. [...]». Et le ministre de poursuivre, certainement sur les ordres sinon sous la dictée de Napoléon : «l'Empereur ne croit plus à l'alliance de la Russie», avant de terminer par ces paroles péremptoires : "Anéantissez cette lettre du moment où vous l'aurez lue, et qu'il n'en reste aucune trace ${ }^{38}$."

Cette dépêche, donc, fut expédiée le 2 juin. Le lendemain, une lettre du prince Kourakin à Aubert, chargé d'affaires de Russie à Vienne, fut interceptée par les services de renseignements français. Elle fut aussitôt transmise à Daru qui la jugea suffisamment importante pour être communiquée non seulement à Champagny, mais aussi, directement, à l'empereur, ce qu'il fit par une lettre autographe datée du 3 juin I809:

Sire,

J'ai l'honneur d'adresser à Votre Majesté la copie d'une lettre de M. le prince Kourakin à M. d'Aubert Conseiller d'Etat de Russie actuellement à Vienne.

Cette lettre qui contient une profession de principes n'étoit point chiffrée \& est arrivée ce soir par la poste.

Je suis avec le plus profond respect

Sire,

De votre majesté i[mpériale] \& R[oyale].

Le très obéissant serviteur $\&$ très fidéle sujet

Vienne ce 3 juin 1809

$\operatorname{Daru}^{39}$

37. AMAE, Correspondance politique, Autriche, Supplément, vol. 28, fo $162 \mathrm{r}^{\circ}$.

38. ASHAT, 2 C 9I, copie.

39. AN, AF IV I677, pl. $2^{\text {III }} n^{\circ} 78, f^{\circ} 66$ I. 
Les termes employés ici par Daru sont significatifs. Andréossy, on vient de le voir, avait employé dans sa dépêche du Is février le même mot "principes", qui revient très souvent dans le contexte des discussions et rapports concernant l'attitude de la Russie. Il fut également employé par Kourakin dans la lettre interceptée, missive à caractère personnel, mais qui contient une allusion à ses principes, qualifiés par le prince lui-même de "conservateurs». Ce qui laissait entendre qu'il ne soutenait pas Napoléon dans sa marche sur Vienne.

Voici donc la copie qui en fut transmise à l'empereur par Daru. Elle est, à notre connaissance, inédite et, on le voit, de la main de Beyle (fig. 13, p. 70) :

Copie d'une lettre adressée à M. d'Aubert Conseiller d'Etat de S. M. l'Empereur de toutes les Russies $\&^{a}$ à Vienne. Datée Paris le 2/24 Avril I809. Signée du Prince Alexandre Kourakin.

J'ai beaucoup regretté, Monsieur, que mon départ de Vienne m’ait laissé si peu jouir du plaisir que m’avait fait votre arrivée. Il aurait été aussi véritable que le sont mes anciens sentimens pour vous, dont j'ai chargé bien particulièrement Freygany de vous porter les expressions. Je n'ai point à me faire le reproche d'avoir épargné les soins et les efforts pour détourner la catastrophe qui va, de nouveau, dévaster le continent. J'ai toujours agi avec la plus grande énergie, dans le sens des principes conservateurs que vous me connaissez, que vous savez apprécier dans mes vues, et dont vous même me paraissez pénétré. - Je ne cherche point à être informé de ceux que peuvent avoir adopté les employés qui m’entourent. Je ne demande d'eux que de faire leur devoir, et de suivre mes ordres, et s'ils ont dans leur conviction d'autres idées que les miennes, tout ce que je puis faire, c'est de les en plaindre. C'est avec beaucoup de plaisir que j'ai appris que votre aimable famille pense toujours à moi, ce qui est de toute justice par les sentimens que je lui conserve, et que je vous prie de témoigner à tous ceux qui la composent, et particulièrement envers votre fils, puisque je ne puis oublier les liens que vous m'avez fait contracter envers lui. Croyez toujours aux sentimens de mon ancienne amitié, et de ma considération parfaite ${ }^{40}$.

C'est également Beyle qui sera chargé d'en faire la copie destinée à Champagny ${ }^{4 \mathrm{I}}$, ainsi que de retranscrire dans le registre de la correspondance active de Daru, la lettre au ministre, datée de même du 3 juin I809, qui l'accompagnait. Celle-ci reproduit le texte de la lettre à Napoléon, à une variante révélatrice près : la lettre de $M$. de Kourakin, y lit-on, "a été trouvée parmi celles examinées ce soir à la poste ${ }^{42}$ ».

Le travail accompli ici par Beyle est, certes, celui d'un secrétaire, mais d'un secrétaire bien au fait des affaires diplomatiques et qui, ne serait-ce que de manière modeste, "tient la plume», formule qu'il emploiera lui-même et sur laquelle nous reviendrons.

40. AN, AF IV I677, pl. $2^{\mathrm{III}} \mathrm{n}^{\circ} 77$, fo 660 .

4I. AMAE, Correspondance politique, Autriche, vol. 384 , fo 126.

42. $\mathrm{AN},{ }^{*} \mathrm{I} 38 \mathrm{AP} 73$. 
Ajoutons auparavant, à l'intention de ceux qu'intéresse l'histoire de la musique, que c'est vraisemblablement dans ce contexte de surveillance que Stendhal a rencontré pour la première fois le nom, sinon la personne, de Giuseppe Carpani, dont il allait piller un des ouvrages, les Haydine ${ }^{43}$, lors de la composition des Vies de Haydn, de Mozart et de Métastase, qu'il jugea bon de publier sous le pseudonyme de Louis-Alexandre-César Bombet ${ }^{44}$.

Protégé de la famille impériale autrichienne, auteur de nombreux livrets commandés par l'impératrice Maria Theresa, Carpani était connu de longue date pour ses opinions hostiles à la France. Les articles anti-français qu'il avait publiés dans la Gazzetta di Milano en I796, l'avaient obligé à s'enfuir à Vienne avec l'archiduc Ferdinand ${ }^{45}$. Au début de la campagne de I809, Carpani était aux côtés de l'archiduc Jean, ce qui ne manqua pas de parvenir aux oreilles de Napoléon. Le 6 avril, l'empereur adressa au prince Eugène de Beauharnais, vice-roi d'Italie, une demande de renseignements conçue en ces termes :

Mon fils, qu'est-ce que c'est qu'un nommé Carpani, poëte, qui est avec l'archi-

duc Jean? Faites surveiller ses parents, et, s'il a des biens, faites-les saisir ${ }^{46}$.

Napoléon avait sans doute déjà eu connaissance d'un bulletin de renseignements transmis au ministre des Affaires étrangères, le 29 mars I809, par l'ambassade de France à Vienne. Il y était question non seulement de Carpani, mais aussi de Schlegel :

$\underline{\text { Bulletin }}$

Vienne, 29 mars I809.

$\mathrm{M}^{\mathrm{r}}$ Carpani, poète et auteur dramatique italien, est nommé secrétaire de l'Archiduc Jean. Il a déjà formé ses dispositions de campagne, il sera chargé de rédiger les bulletins et les proclamations. Cet individu est l'ami de la Maison Ferdinand, où il va tous les jours. Il se vante d'avoir élevé l'impératrice régnante. Carpani est l'âme de la société anti-française. Il y plait par ses propos contre la France et ses contes du plus mauvais goût. Il est Milanais.

43. Le Haydine ovvere lettere sulla vita e le opere del celebre maestro Giuseppe Haydn, Milan, Buccinelli, I8I2.

44. Titre de la première édition publiée à Paris par P. Didot l'Aîné au début de I8I5 (avec le millésime 1814) : Lettres écrites de Vienne en Autriche, sur le célèbre compositeur J ${ }^{H}$. Haydn, suivies d'une vie de Mozart, et de considérations sur Métastase et l'état présent de la musique en France et en Italie.

45. Voir J. Haydn, Autobiographie, premières biographies, traduites et présentées par M. Vignal, Flammarion, coll. «Harmoniques», 1997, p. I6.

46. E. de Beauharnais, Mémoires et correspondance politique et militaire du prince Eugène, t. IV, publiés, annotés et mis en ordre par A. du Casse, Michel Lévy frères, I858-I860, p. 426. 
Le Prince Charles a nommé son secrétaire, $\mathrm{M}^{\mathrm{r}}$ Schgel [sic], homme de lettres estimé par son érudition. Le Prince Charles lui donne des appointemens considérables et la charge d'écrire ses campagnes.

Ces deux choix font beaucoup parler ici; on est étonné de voir des étrangers jouir d'une telle confiance, et un histrion placé auprès d'un grave Prince qui est plus instruit en mathématiques qu'en toute autre science, et un savant auprès d'un général qui ne connaît absolument que son métier $[\ldots]^{47}$.

Ces propos, on le devine, n’auraient pas été pour déplaire à celui que Carpani allait nommer quelques années plus tard "ce fils de l'Isère écumante ${ }^{48}$ " - notre Louis-Alexandre-César Bombet.

On peut se demander, par ailleurs, si ce n'est pas également dans ce contexte du renseignement que Beyle avait été chargé de la mission en Hongrie qu'il évoque, en termes très vagues, dans une lettre adressée le 25 juillet à sa sœur Pauline ${ }^{49}$. Plusieurs thèses ont été avancées à ce propos : on y a vu une mission d'espionnage, de reconnaissance des mouvements des troupes ennemies; une mission de recherche d'approvisionnements ou d'objets d'art; ou encore une mission diplomatique.

Passons ces thèses en revue.

S'agit-il d'une mission d'espionnage? Les services de renseignements étaient, on le sait, très développés sous l'Empire. Daru, comme nombre de ministres et administrateurs, avait déjà été mis à contribution dans ce genre d'opération. Ainsi, lorsque Napoléon reçut à Bayonne, le I ${ }^{\text {er }}$ juillet I808, des renseignements sur le réarmement de l'Autriche, qui le préoccupait depuis quelques mois déjà, il fit donner à Daru et au maréchal Davout l'ordre $\mathrm{d}^{\prime}$ ' "envoyer des agents en Bohème pour connaître les mouvements réels des Autrichien $s^{5 \circ}$ ». Une mission de ce genre a-t-elle été confiée à Beyle? A-t-il été chargé de vérifier les mouvements de l'armée autrichienne en Hongrie, de concert éventuellement avec les services du prince Eugène, qui envisageait en juillet I809 d'établir à Eisenstadt son quartier général, alors installé à Raab? Ce n'est pas impossible, mais aucun document n'est venu jusqu’à présent le prouver.

Deuxième explication proposée : la recherche de vivres et de nouvelles montures pour l'armée. À l'appui des arguments déjà mis en avant à ce sujet,

47. AMAE, Correspondance politique, Autriche, vol. $382, f^{\circ} 217 \mathrm{r}^{\circ}$.

48. Le Majeriane, ovvero lettere sul bello ideale di Giuseppe Carpani in risposta al libro Della imitazione pittorica del cav. Andrea Majer, p. 2I4. Traduction de D. Muller, OC, t. XLI, Padoue, I824, p. 490.

49. $C G$, t. I, p. 838 .

50. CN, t. XVII, p. 343, lettre au prince de Neuchâtel. 
notamment par Arthur Chuquet et Richard Coe ${ }^{\text {sI }}$, on peut proposer quelques éléments complémentaires. La correspondance de Napoléon est rarement citée par les biographes de Stendhal, mais la lecture des lettres et ordres de l'empereur, ainsi que celle de documents d'archives encore inédits, semblerait conforter l'hypothèse selon laquelle Beyle serait parti chercher ou demander des vivres, fourrages, bœufs ou chevaux en Hongrie.

Le 24 juin I809, en effet, l'empereur avait écrit au comte Daru :

Monsieur Daru, il y a à Neusiedler ${ }^{r_{2}}$ vingt mille sacs de grains qui appartenaient à l'archiduc Albert. Vous pouvez les faire prendre pour l'approvisionnement de Viennes.

Napoléon s'était également adressé au prince Eugène, à plusieurs reprises, pour lui demander de diriger sur Vienne le blé, la farine, les bœufs qui manquaient dans la capitale. Et le 29 juin, il dut insister : "Mon Fils, envoyeznous donc des vivres; nous sommes dans la plus grande pénurie. [...] Nos besoins en vivres sont tout à fait pressants ${ }^{54}$." Aux bureaux de l'intendance générale de l'armée, cette pénurie fit l'objet d'une correspondance abondante, en partie de la main de Beyle qui, quelques années plus tard, dans sa Vie de Napoléon, allait qualifier de "chef-d'œuvre» le travail accompli par Daru en ce domaine. «Mais, ajoutera-t-il, l'on ne parla pas de ce tour de force, car il eût fallu avouer le dangers.» Était-ce donc là le véritable objet de sa mission? Était-ce pour ne pas "avouer le danger» auquel était exposée l'armée, qu’il ne révéla pas à Pauline la raison pour laquelle il s'était rendu en Hongrie? Encore une fois, c'est possible, mais rien ne permet de l'affirmer de manière catégorique. Nous n'avons que des présomptions, assez fortes il est vrai.

Il en est de même de la recherche d'objets d'art. Mais là, les présomptions sont, à notre sens, moins fortes. Certes, au début des hostilités, beaucoup d'objets précieux avaient été mis en sûreté en Hongrie. Ils pouvaient échapper ainsi, en juillet I809, à la vigilance de Vivant Denon, qui réunissait alors des œuvres destinées à compléter les collections du musée Napoléon et de la bibliothèque impériale. Mais à cette époque il avait déjà accompli l'essentiel de son travail de recensement et des marchés avaient été passés, le is juillet, pour l'emballage et le transport des caisses de Vienne à Paris ${ }^{56}$.

51. A. Chuquet, Stendhal-Beyle, Plon-Nourrit et $\mathrm{C}^{\mathrm{ie}}$, 1902 [2 $2^{\mathrm{e}}$ éd.]; R. N. Coe, «Lisbeth folle par amour ou les soirées d'un commissaire des guerres", Stendhal Club, n ${ }^{\circ} 39$, I5 avril I968, p. 255

52. Localité inscrite sur l'itinéraire hongrois de Beyle, qui évoque le Neusiedler See (lac de Neusiedl) dans sa lettre du 25 juillet I809 à Pauline, CG, t. I, p. 838 (Voir «Dossier», p. I75).

53. CN, t. XIX, p. I68.

54. Ibid., p. 194.

55. Vie de Napoléon, OC, t. XXXIX, chap. XXXV, p. I20.

56. ASHAT, 2 C 95, état de dépenses signé par Denon, $\mathrm{I}^{\mathrm{er}}$ août $\mathrm{I} 809$. 
Examinons enfin l'hypothèse de la mission diplomatique. La première fois que Beyle évoque son voyage en Hongrie, dans la lettre adressée à sa sœur le 25 juillet, il reste très discret :

Dernièrement, j'ai été chargé d'une mission en Hongrie; je me suis promis en sortant de Vienne de ne plus songer pendant vingt-quatre heures à ce qu'il renfermait. C'était peut-être la seule occasion de ma vie que j'avais de voir cette célèbre Hongrie ${ }^{57}$.

Qu'y voit-il, justement? Il en dit très peu à Pauline, et évoque bien laconiquement son passage à Eisenstadt, résidence des Esterhazy qui pourrait être, cependant, une des pièces clés de notre puzzle : «De Laxenbourg, écrit-il, j'allai à Eisenstadt et, de là, aux abords du $\operatorname{lac}^{58}$ que tu verras sur les cartes. » Suivent quelques observations sur le costume croate mais, sur la mission, aucune précision. Serait-ce la preuve qu'il s'agissait d'une mission hautement secrète? S'agissait-il de ce qu'il qualifia lui-même, plus de vingt ans plus tard, d' "affaire de la Hongrie, la plus singulière de l'époque», c'est-à-dire, de «donner la Hongrie à un archiduc ${ }^{59}$ "?

Stendhal mentionne à plusieurs reprises dans ses écrits le projet qu'aurait eu Napoléon d'offrir la couronne de Hongrie à l'archiduc Charles, ou au grand-duc de Würzburg. Et il n'affabule pas. Ces projets sont en effet très bien documentés.

Napoléon avait d'abord envisagé de séparer les trois couronnes d'Autriche, Bohême et Hongrie, et d'offrir la couronne de Hongrie à un prince de ce pays. Le I5 mai I809, deux jours après son entrée dans Vienne, il fit publier une proclamation dans ce sens :

Hongrois! Le moment est venu de recouvrer votre indépendance. Je vous offre la paix, l'intégrité de votre territoire, de votre liberté et de vos constitutions [...]. Vous avez des mœurs nationales, une langue nationale; vous vous vantez d'une illustre et ancienne origine : reprenez donc votre existence comme nation. Ayez un roi de votre choix, qui ne règne que par vous, qui réside au milieu de vous, qui ne soit environné que de vos citoyens et de vos soldats. Hongrois! Voilà ce que vous demande l'Europe entière qui vous regarde; voilà ce que je vous demande avec elle ${ }^{60}$.

Peu de contemporains ignoraient que le candidat pressenti était le prince Esterhazy, dont une des résidences d'été se trouvait, précisément, à Eisenstadt. Un rapport de renseignements sur la cour de Vienne et la Hongrie qui est conservé aux archives du Quai d'Orsay laisse entendre que l'intention exprimée

57. $C G$, t. I, p. 838 .

58. Il s'agit du lac de Neusiedl. Voir note 52 supra.

59. États de service joints à la lettre du 25 août I830 au comte Molé, CG, t. III, p. 773.

6o. $C N$, t. XIX, p. II-I2. 
par Napoléon n'était pas contraire aux ambitions du prince : l'auteur anonyme y affirme que ce dernier, chez qui il venait de passer deux jours à Eisenstadt, s'était «entretenu avec [lui] avec assez d'abandon" au sujet de l'avenir du pays ${ }^{6 \mathrm{~T}}$.

Mais Napoléon avait également envisagé, nous l'avons dit, de placer l'archiduc Charles sur le trône, ou alors, afin de maintenir l'intégrité de l'Autriche, de faire abdiquer l'empereur François II en faveur du grand-duc de Würzburg. Le Io septembre 1809 , il réaffirmait ces intentions dans une lettre adressée à Champagny à propos d'une conversation qu'il venait d'avoir avec l'aide de camp de l'empereur d'Autriche, le comte de Bubna :

J'ai dit que [...] s'il y avait un empereur à la bonne foi duquel je pusse me fier, comme le grand-duc de Würzburg ou l'archiduc Charles, je rendrais toute la monarchie autrichienne et je n'en retrancherais rien. L'aide de camp m’a répondu que si l'empereur était persuadé de cette vérité, il abandonnerait le trône pour y placer le grand-duc de Würzburg. Je lui ai dit que j’avais déjà fait cette proposition à M. de Liechtenstein ${ }^{62}$.

Le I5 septembre i809, Napoléon adressa à Champagny une longue lettre au sujet d'une nouvelle conversation qu'il avait eue avec Bubna et pendant laquelle il avait renouvelé ses propositions concernant la couronne hongroise :

$\mathrm{M}^{\mathrm{r}}$. de Bubna m'a parlé, de la part de l'Empereur, du chagrin que lui donnent les conférences d'Altenburg, et du mécontentement que cela lui donnoit contre $\mathrm{M}^{\mathrm{r}}$. de Metternich. Je l'ai confirmé dans ce mécontentement en disant [...] qu'ils avoient fait une grande faute en mécontentant le prince Charles, qui est leur meilleur général; que le prince de Liechtenstein est une tête d'alouette; que le général Bellegarde ne voit pas clair; mais que le prince Charles est un homme sage, qui étoit aimé de leurs troupes et avoit leur confiance. J'ai ajouté que [...] mon véritable intérêt étoit de séparer les trois couronnes, ou de faire une alliance intime avec la maison régnante; que la séparation des trois couronnes n'étoit proposable qu'en se battant encore; et que c'étoit une fâcheuse extrêmité que de se battre et répandre encore du sang; que l'intime alliance avec l'Empereur actuel étoit difficile, parce que, quoique rempli de bonnes qualités, il est toujours de l'opinion du dernier qui lui parle [...]; que j'avois dit au prince de Liechtenstein, que je l'avois dit et répété à qui vouloit l'entendre, que l'Empereur cède le trône au grand-duc de Würzbourg, je restitue tout à l'Autriche sans rien exiger; que, cependant, je sentois que cette proposition n'étoit pas fesable. Mr. de Bubna m'a arrêté là, en me disant que l'Empereur n'étoit pas éloigné de faire ce sacrifice, puisqu'il étoit utile à ses peuples. Je lui ai répondu que je l'accepterois; que la base mise en avant aux négociations d'Altenburg n'étoit pas de rigueur; que l'uti-possidetis étoit pire que la séparation des trois couronnes et destructif de la monarchie [...]. - Vous comprenez, donc, le sens et l'esprit de ma conversation. Faites-la connoître à $\mathrm{M}^{\mathrm{r}}$. de Metternich pour qu'il n'en ignore pas; et insinuez-

6I. AMAE, Correspondance politique, Autriche, vol. 383 , fo $343 \mathrm{v}^{\circ}-344 \mathrm{r}^{\circ}$. Napoléon a écrit en haut de la première page de ce rapport : "Renvoyer à Champagny».

62. $C N$, t. XIX, p. 447. 
lui que, si l'Empereur actuel vouloit laisser le trône au grand-duc de Würzbourg, pour une raison quelconque (on dit, par exemple, qu'il est dégoûté), je laisserois entière la monarchie. $[\ldots]^{63}$.

Ce texte confirme ainsi les observations de Beyle-Stendhal au sujet de la Hongrie. Il soulève également, par le biais des allusions à l'«alliance intime» qui lierait la France et l'Autriche, la question de l'éventuel mariage de Napoléon avec l'archiduchesse Marie-Louise, déjà l'objet de discussions à l'automne I809, bien avant que le divorce avec Joséphine ne soit prononcé. Nous y reviendrons.

Voilà donc où en sont les affaires franco-autrichiennes en juillet - septembre I809. Stendhal évoquera la question hongroise, nous l'avons dit, dans la Vie de Napoléon, en I817-1818 :

Napoléon, frappé de la bravoure des Hongrois et se souvenant de leur esprit national, eut quelque velléité de faire de la Hongrie un royaume indépendant; mais il craignit de négliger l'Espagne, et, d'ailleurs, il ne vit jamais toute l'étendue de cette idée ${ }^{64}$.

Il y fera également allusion, à deux reprises, dans Rome, Naples et Florence :

[...] Napoléon était à Vienne, où il gagnait tout juste la bataille de Wagram; l'Espagne l'inquiétait; il songeait à donner la Hongrie à l'archiduc Charles, etc. ${ }^{65}$

[Napoléon] manquait d'une autre qualité principale du politique : il ne savait pas saisir l'occasion qui souvent n'existe que pendant quelques heures. Par exemple, pourquoi, en I809, ne pas donner le royaume de Hongrie à l'archiduc Charles, et, en I813, dix millions à M. de $\mathrm{M}^{* * * 66}$ ?

Mais Beyle, avait-il joué un rôle dans cette affaire? Daru l'a-t-il réellement chargé «à Vienne, en 1809 , de tout le travail préparatoire à la formation du royaume de Hongrie»? C'est ce qu'il prétend dans un document qu'il aurait adressé en 1833 au duc de Broglie ${ }^{67}$. Peut-on le croire, aussi, lorsqu'il affirme en I830, dans une note autographe résumant ses états de service :

A Vienne, à Berlin, il avait été chargé par M. le comte Daru, son parent, de sa correspondance diplomatique. A Vienne, en I809, M. Beyle tint la plume dans

63. AMAE, Mémoires et Documents, France, vol. I783, fo 6I-62. CN, t. XIX, p. 474475; voir aussi p. 485-486, lettre à Champagny du 2I septembre I809 (l'original, conservé aux archives du ministère des Affaires étrangères, est daté du 20 : Mémoires et Documents, France, vol. 1783, fo 67-68).

64. Vie de Napoléon, $O C$, t. XXXIX, chap. xxxv, p. I2I.

65. Rome, Naples et Florence (I826), dans Voyages en Italie, éd. V. Del Litto, Gallimard, coll. «Bibliothèque de la Pléiade», 1973, p. 397.

66. Ibid., p. 424.

67. L. Farges, Stendhal diplomate, Rome et l'Italie de I829 à 1842 d'après sa correspondance officielle inédite, Plon, Nourrit et $\mathrm{C}^{\mathrm{ie}}$, Paris, 1892, p. 225; CG, t. IV, p. 717. La localisation actuelle de ce manuscrit est inconnue. 
toute l'affaire de la Hongrie, la plus singulière de l'époque. Il s'agissait de donner la Hongrie à un archiduc ${ }^{68}$ ?

Cette affirmation laisse Arthur Chuquet incrédule : "Comme si Daru, s'exclame-t-il, eût proposé son étourdi de cousin, un simple adjoint aux commissaires des guerres, pour une négociation de cette importance ${ }^{69}$ !»

Notons en passant que Beyle ne prétend pas avoir été chargé de la négociation. Il affirme simplement avoir tenu la plume dans le travail préparatoire à la formation du royaume de Hongrie. Et nous avons vu que Daru l'avait précédemment chargé d'un travail préparatoire important, en mars I809 : celui effectué dans de cadre de la composition de la liste civile en Toscane. En outre, l'affaire Kourakin, évoquée plus haut, nous apporte la preuve que Daru l'avait bien chargé à Vienne de "tenir la plume» dans un contexte diplomatique. Par ailleurs, précisons-le d'ores et déjà, on retrouve la trace de sa plume dans la correspondance relative aux articles secrets du traité de paix d'octobre I809, et dans une lettre de Daru à Napoléon concernant les convois d'argent destinés à l'acquittement des contributions de guerre de l'Autriche lors du départ des troupes françaises ${ }^{70}$.

Ces considérations justifient, à notre sens, les déclarations de Beyle. François Michel, qui avait mené des recherches approfondies aux archives du ministère des Affaires étrangères, était persuadé que Beyle "a[va]it été mêlé" au «travail sur la création d'un Royaume indépendant de Hongrie ${ }^{71}$ ». Et il semble en avoir convaincu Henri Martineau, d'abord dubitatif, et Victor Del $\mathrm{Litto}^{72}$. À l'appui de sa thèse, nous apporterons un dernier argument.

Louis Farges a présenté comme étant adressé au duc Victor de Broglie, ministre des Affaires étrangères du II octobre I832 au I3 avril I834, le document où Stendhal affirmait que Daru lui avait confié «tout le travail préparatoire à la formation du royaume de Hongrie ${ }^{73}$ ». Or, en juillet I809, le futur ministre, pour lors jeune auditeur au Conseil d'État attaché au service de l'intendant général de l'armée d'Allemagne, se trouvait à Vienne, où il avait

68. L. Farges, ouvr. cité (note 67 supra), p. 275; CG, t. III, p. 773. Farges date cette note (dont la localisation est aujourd'hui inconnue) de septembre i830, alors que H. Martineau la considère comme une pièce jointe à la lettre du 25 août I830 au comte Molé (Correspondance, t. VI, Le Divan, I933-I934, p. 3II, note I).

69. A. Chuquet, ouvr. cité (note sI supra), p. Ioo.

70. Nous publierons ces textes dans une édition critique actuellement en préparation.

7I. Fr. Michel, «Deux ministres et un consul. Le comte Molé, le duc de Broglie et Stendhal ", ouvr. cité (note 26 supra), p. 312 note 2.

72. Stendhal, Correspondance, éd. H. Martineau et V. Del Litto, coll. «Bibliothèque de la Pléiade», Gallimard, 1962-1968, t. I, p. 536 note 2 : «[...] il est croyable que la mission qu'accompagnait Stendhal allait faire à un prince de cette Maison [les Esterhazy] des ouvertures pour lui offrir le trône de Hongrie».

73. L. Farges, ouvr. cité, loc. cit. (note 67 supra). 
été envoyé quelques jours après la bataille de Wagram ${ }^{74}$. Il a dû y rencontrer Beyle chez le duc de Bassano et le comte Daru qui "tenaient table ouverte " $^{75}$ " pour les auditeurs, et semble avoir partagé son avis quant au caractère de l'intendant général :

$[\ldots]$ je n'ai $[\ldots]$ eu qu'une très faible part dans les réprimandes que $M$. Daru n'épargnait pas à ses jeunes collaborateurs. [...] je ne sortis point des limites du territoire dont l'administration m'était confiée, et, moyennant cette précaution, j'évitais les reproches que j'aurais reçus, sans doute, bien ou mal à propos, si j'avais montré mon visage, et donné occasion de penser à $\mathrm{moi}^{76}$.

C'est à Daru lui-même qu'il dut la faculté de prendre cette mesure préventive. L'intendant général allait en effet le nommer intendant du comitat de Raab-Eisenbourg, qui était situé en Hongrie ${ }^{77}$. Cela étant, est-il concevable que Beyle, consul de France à Civitavecchia lorsqu'il évoque, en I833, son travail pendant la Campagne de Vienne de I809, ait menti à son ministre, alors que celui-ci était des mieux placés pour lui donner le démenti? Même un étourdi comme Beyle aurait compris que Broglie ne manquerait pas de dévoiler l'imposture.

Pour toutes ces raisons, il nous semble possible d'accepter les affirmations de Stendhal, sans outrer le portrait d'un "simple adjoint aux commissaires des guerres» qui a su mériter - quoiqu'il en dise dans son journal - quelques égards de la part de Daru, comme le pensait François Michel.

Abordons, enfin, la question de savoir si Beyle a pris part aux premières négociations du mariage de Napoléon et de l'archiduchesse Marie-Louise. Son cousin Romain Colomb écrit à ce propos :

Tout en faisant une rude guerre à l'Autriche, Napoléon, pendant son séjour à Vienne, ne perdait pas de vue ses projets de mariage avec l'archiduchesse MarieLouise. Beyle, dont la capacité et la discrétion avaient pu être appréciées dans maintes circonstances, participa aux travaux et aux négociations qui précédèrent ce grand événement ${ }^{78}$.

Cette alliance, nous l'avons vu, faisait en effet l'objet de négociations pendant le séjour de Napoléon à Vienne, et cela dès le mois de septembre i809. Si Beyle a été associé à celles concernant la couronne de Hongrie, il n'est pas impossible qu'il ait également "tenu la plume» pendant des réunions où

74. V. de Broglie, Souvenirs, I785-I870, publiés par son fils le duc C.-J.-V.-A. De Broglie, Calmann Lévy, I886, t. I, p. 7I.

75. Ibid., p. 73.

76. Ibid., p. 78-79. Broglie avait néanmoins qualifié Daru de «bon, aimable, ouvert» dans une série de portraits des membres du Conseil d'État impérial (ibid., p. 64).

77. Ibid., p. $75-84$.

78. «Notice sur la vie et les ouvrages de Henri Beyle (de Stendhal)», OC, t. XLIX, p. 259. 
il était question de l'archiduchesse. Rien, toutefois, en l'état actuel de nos recherches, ne permet de l'affirmer. Il existe, en revanche, une page de notes inédites autographes qui est conservée aux Archives nationales parmi les documents relatifs aux cérémonies du mariage en avril I8Io (fig. I4, p. 7I) :

L'empereur couchera à St. Cloud le samedi.

Le Dimanche L.L. MM. Entendront

la messe à $S^{t}$. Cloud à Io heures du matin

Après la messe LL. MM. se rendront

à Paris.

A midi L'Empr. partira pour le

Corps législatif

SM. reviendra aux tuileries à 8 heures

banquet.

Après le banquet Concert sur la

terasse.

Après le Concert Spectacle

Après le Spectacle on rentrera dans

les appartemens, où il y aura cercle

un moment, et immediatement

on reviendra à $\mathrm{S}^{\mathrm{t}}$. Cloud ${ }^{79}$.

Comme le mariage eut lieu en I8Io, et que cela nous oblige à quitter notre année 1809 , nous nous limiterons ici à quelques observations rapides. Précisons tout d'abord que les événements présentés ici par Beyle ne correspondent pas exactement au programme définitif. Il ne faut sans doute pas s'en étonner. Le grand maître des cérémonies, le comte de Ségur, avait dû régler en très peu de temps le cérémonial de ce mariage dont le contrat provisoire n'avait été ratifié à Vienne que le i 6 février. Stendhal a ironisé dans les Souvenirs d'égotisme sur le programme "inventé» par Ségur. C'était, selon lui, un "labyrinthe de petitesses ${ }^{80}$ ». Même les flatteurs de la cour de l'empereur ne purent s'empêcher d'en rire, ce qui a pu constituer un des motifs des réaménagements successifs imposés au grand maître. Celui-ci se vit contraint en effet, le 24 mars, de prier Fouché, ministre de la Police générale, d'empêcher la publication des programmes qui lui avaient été envoyés et de défendre aux journaux d'en parler jusqu'à nouvel ordre ${ }^{81}$. Le mariage, initialement fixé au dimanche 25 mars, n'eut lieu, finalement que les $\mathrm{I}^{\text {er }}$ et 2 avril.

Les différences constatées entre le programme définitif et celui présenté par Beyle s'expliquent donc peut-être par les multiples changements apportés au projet initial. Les documents conservés aux Archives nationales permettent

79. $\mathrm{AN}, \mathrm{O}^{2} \mathrm{I} 56, \mathrm{n}^{\circ} 62$.

8o. $O I$, t. II, p. 449.

8I. $A N, F^{7} 3458 n^{\circ} 267$. 
d'en suivre l'évolution au cours du mois de mars I8ı et l'on peut en inférer que la note de Beyle date du début du mois, probablement du 7 ou du 8, c'est-à-dire pendant la première phase des préparatifs.

Certains éléments de son résumé, cependant, recoupent ceux du programme des fêtes organisées, en juin I8II, à l'occasion de la célébration du baptême du roi de Rome. Mais on constate que, dans l'ensemble, le texte de Beyle ne correspond pas davantage au programme définitif de ces célébrations qu’à celui du mariage. Ainsi, qu'il s'agisse du mariage ou du baptême, on est obligé en l'état actuel de nos connaissances, de conclure qu'ici encore Beyle n'a fait que "tenir la plume».

Or, c'est précisément cela - l'écriture, la rédaction - qui nous intéresse chez Stendhal. Pendant la campagne d'Autriche, on a pu le constater, ses activités ne se sont pas limitées à l'accomplissement des tâches subalternes d'un simple adjoint aux commissaires des guerres, comme la recherche de logements, la distribution de dépêches, les secours apportés aux malades. Elles se sont au contraire étendues à la rédaction de documents confidentiels, aux travaux préparatoires aux actes administratifs. C'était une de ses qualités essentielles, capitales, que d'être déjà un excellent rédacteur, intelligent, clair, précis. Ce que soulignera Daru, l'année suivante, en le recommandant au duc de Bassano, secrétaire d'État, comme étant «très propre à rédiger avec netteté, esprit et précision ${ }^{82} »$. C'est sans doute cela qui lui valut en I809 les égards de Daru, constatés par Colomb, devinés par François Michel. C’est certainement ce qui allait lui permettre enfin, en août I8Io, de réaliser son ambition d'être nommé auditeur au conseil d'État. Et sa nomination à ce poste ouvrira pour lui une autre période de grands travaux préparatoires - de projets de rapports ou de décrets qui seront présentés à Napoléon, approuvés et signés par lui ${ }^{83}$ - dont on sait désormais qu'il est possible d'attribuer la composition à Beyle.

\section{Ouvrages cités en abrégé :}

AMAE Archives du ministère des Affaires étrangères, Paris.

AN Archives nationales, Paris.

ASHAT Archives du Service historique de l'armée de terre, Vincennes.

BMG Bibliothèque municipale de Grenoble, Fonds Stendhal.

CG Stendhal, Correspondance générale, éd. V. Del Litto, avec la collaboration d'E. Williamson, J. Houbert et M.-E. Slatkine, Honoré Champion, 6 vol., I997-I999.

82. $O I$, t. I, p. 6I4.

83. Voir E. Williamson, ouvr. cité (note I3 supra). 
Recherches \& Travaux $-\mathrm{n}^{\circ} 79$

CN Napoléon I ${ }^{\text {er }}$, Correspondance de Napoléon Ier, publiée par ordre de l'Empereur Napoléon III, H. Plon et J. Dumaine, 32 vol., I858-1870.

OC Stendhal, Euvres complètes, dir. V. Del Litto et E. Abravanel, Genève, Cercle du Bibliophile, 50 vol., I967-1974.

OI Stendhal, Euvres intimes, éd. V. Del Litto, Gallimard coll. «Bibliothèque de la Pléiade», 2 vol., I98I-I982. 


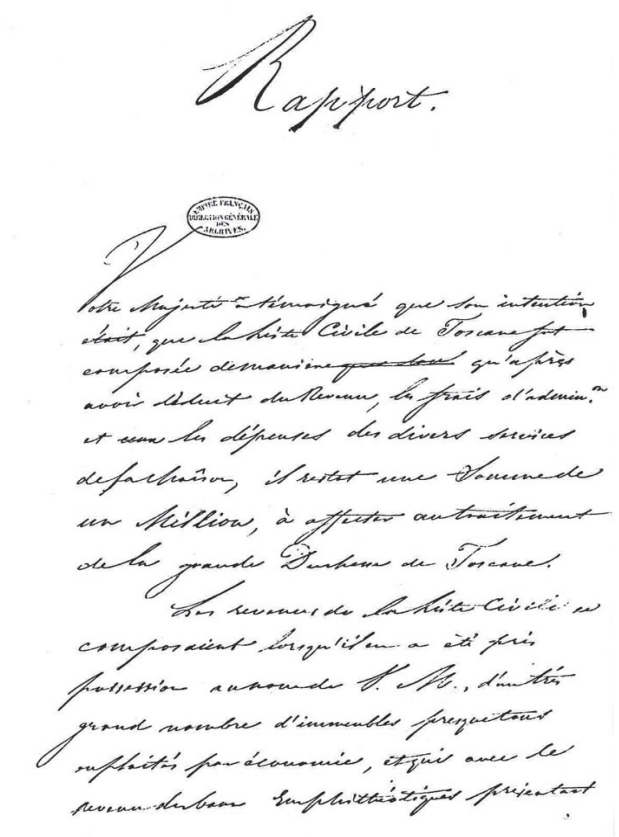

$12:$

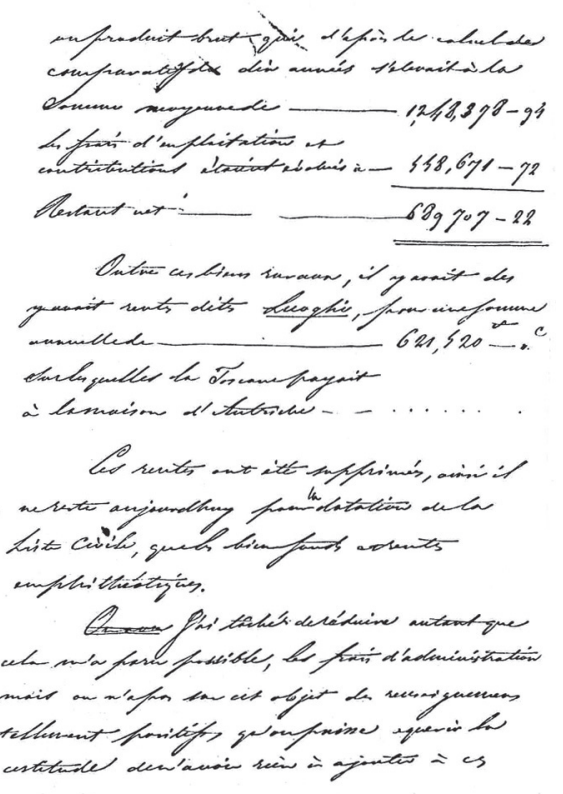

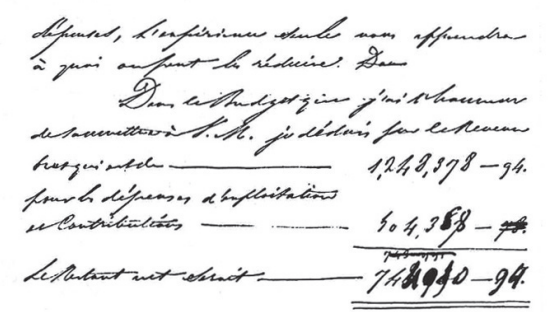

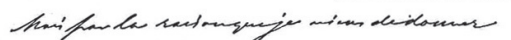

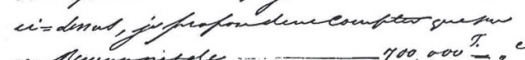

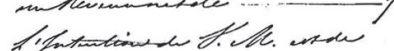

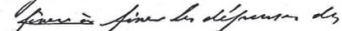

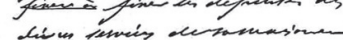

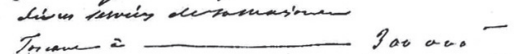

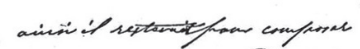

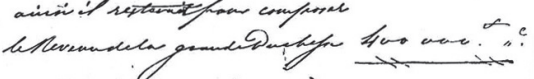

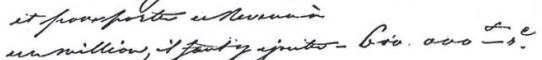

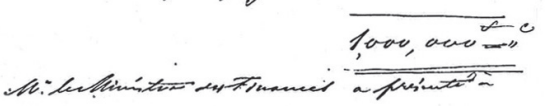

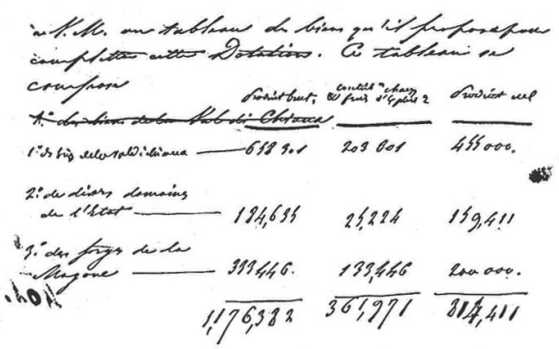

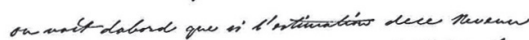

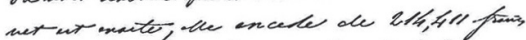

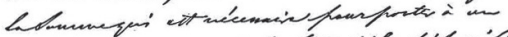

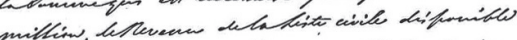

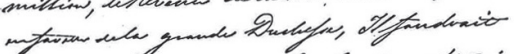

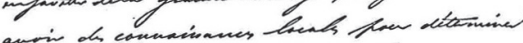

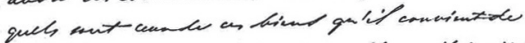

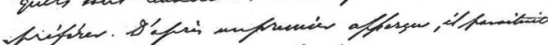

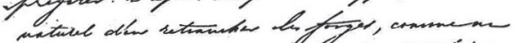

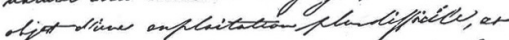
Litur kevenn the variable suevent ts

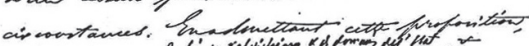

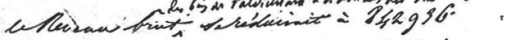

bresugsio $\frac{238525 .}{614,41 !^{\prime 2}}$

Fig. I. Rapport de Daru à Napoléon sur la liste civile de Toscane, projet de la main de Beyle, quelques additions et corrections de la main de Daru, 8-9 mars I809.

$\mathrm{AN}, \mathrm{O}^{2}$ I050, $\mathrm{n}^{\circ} \mathrm{I2}$. 
b.

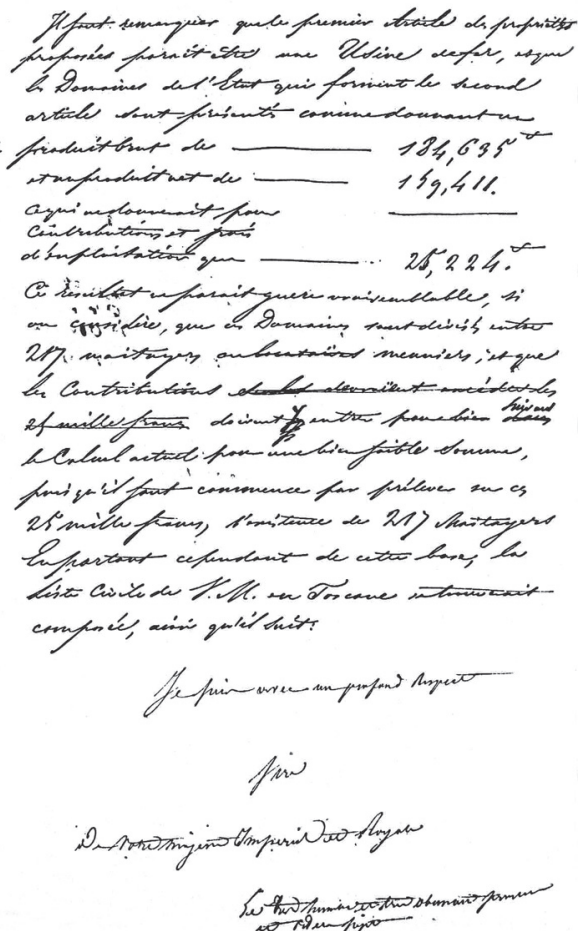

6.
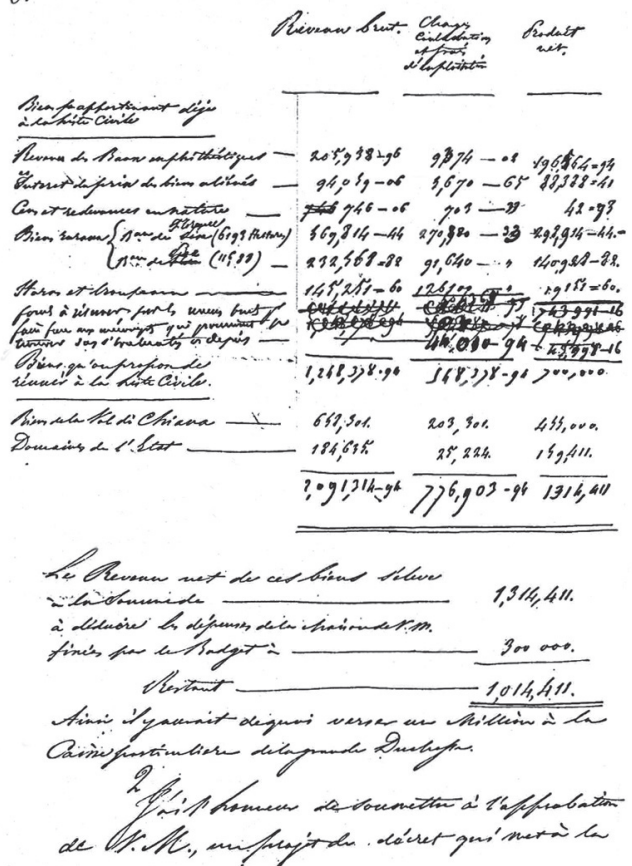

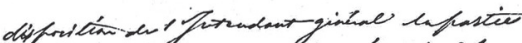

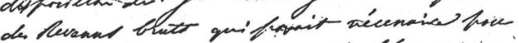
aquiter le Conlsitution arts frás dingliaitation aquaman's as la dorican.

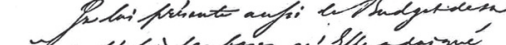

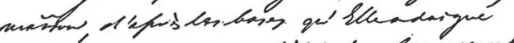

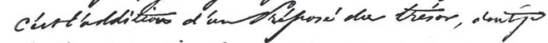

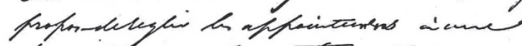

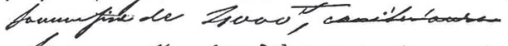

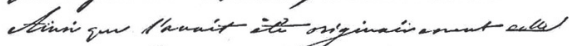

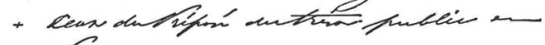
liénant. Niter berions

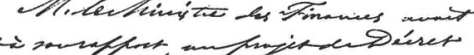

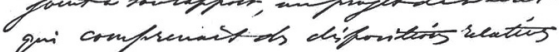

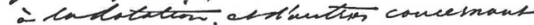

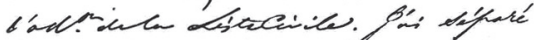

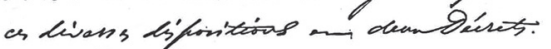
Deper siglaceger at relatif a

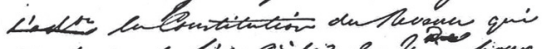

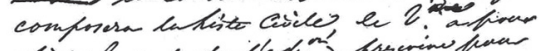

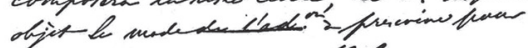
radisule maison de tidl.

Fig. I. (suite) 


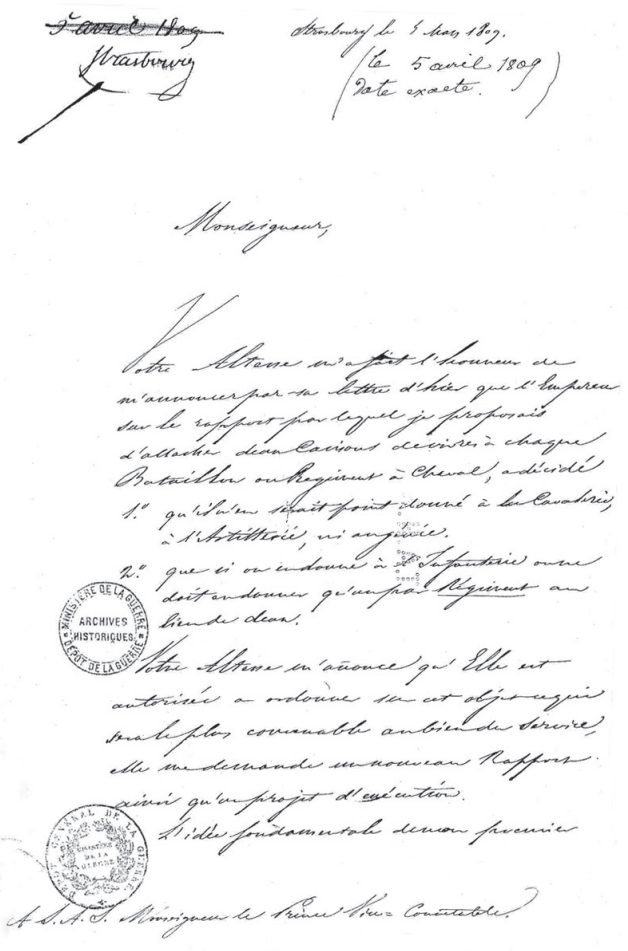

90

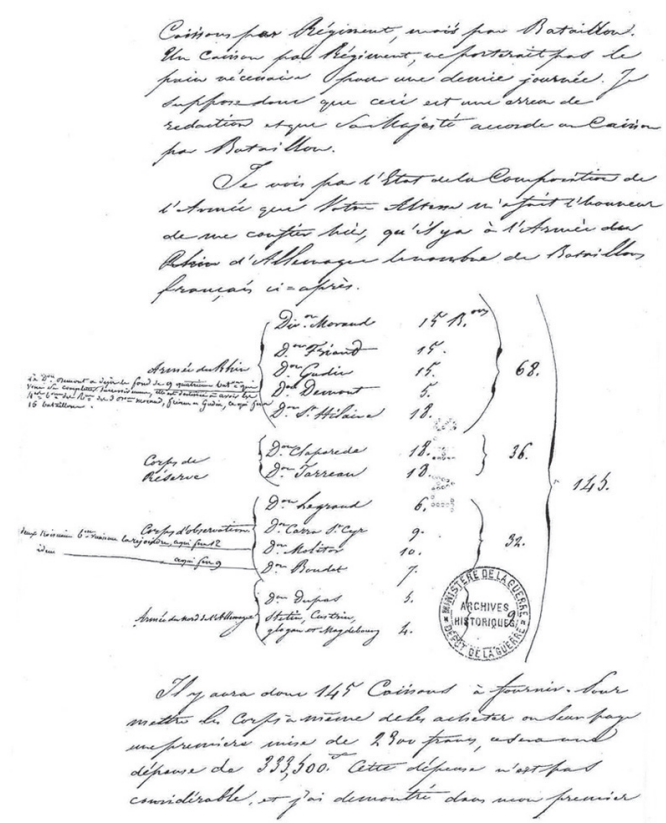

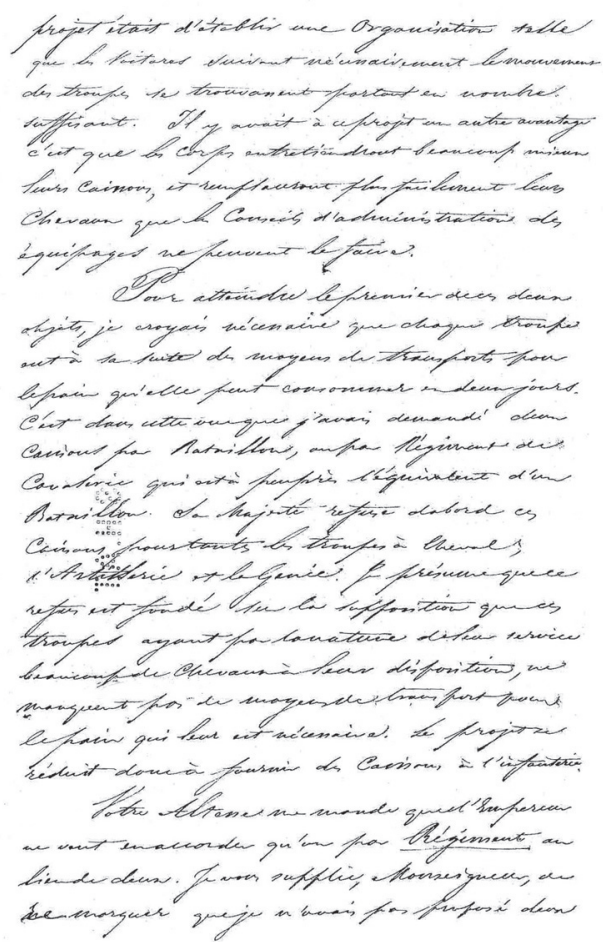

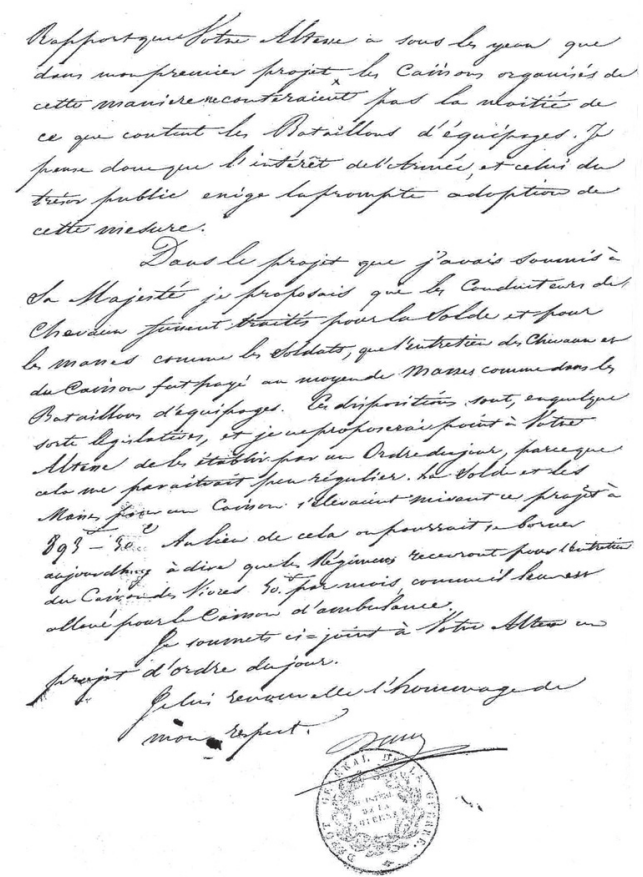

Fig. 2. Lettre de Daru à Berthier de la main de Beyle, signature de la main de Daru, 5 avril I809. ASHAT, 2 C 87. 


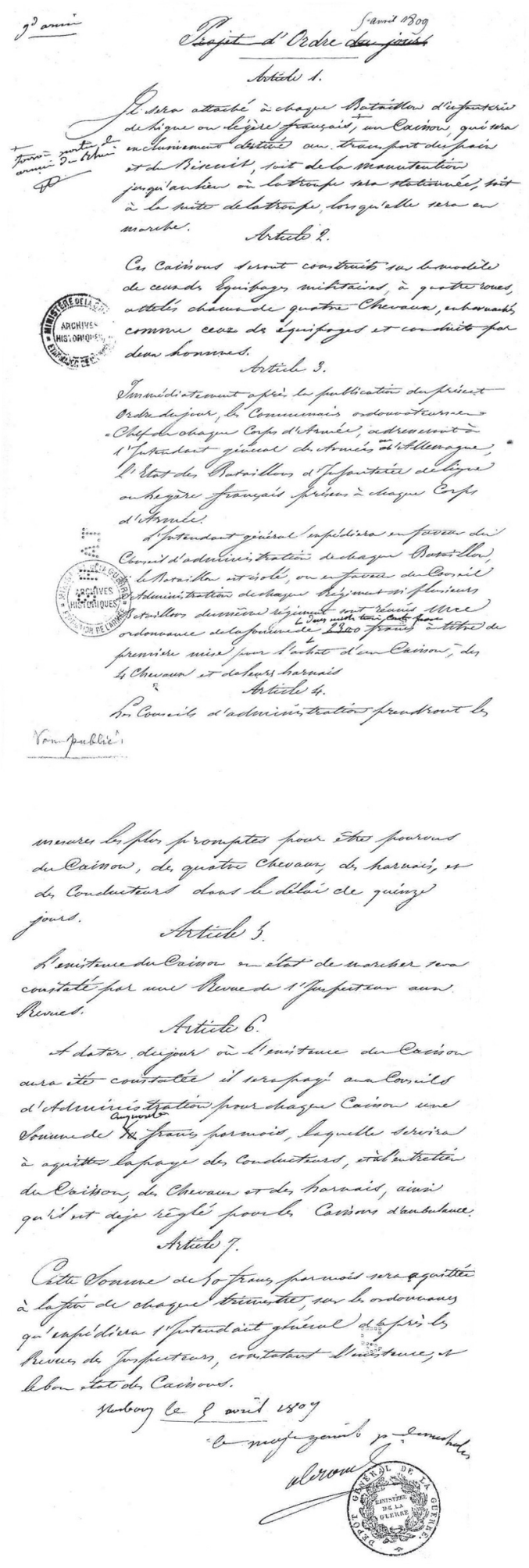

Fig. 3. Projet d'ordre du jour de la main de Beyle, paraphe, signature et quelques ajouts de la main de Berthier, 5 avril I809 (pièce jointe à la lettre précédente, fig. 2). ASHAT, i7 C 82. 

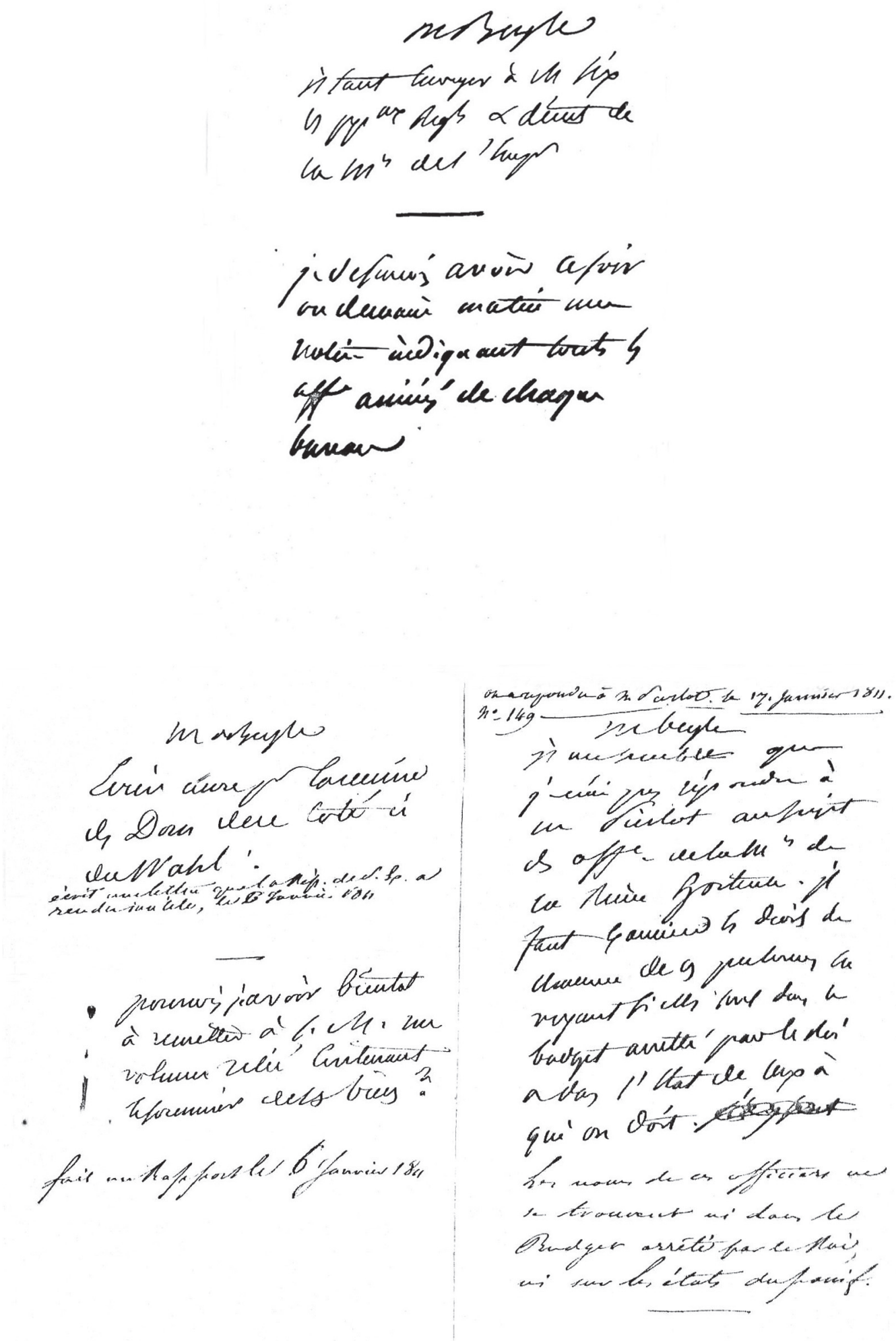

Fig. 4. Billets autographes, griffonnés sur des feuilles volantes, de Daru à Beyle, s. d. [2I-24 novembre I8IO, début et mi-janvier I8II]. Apostilles autographes de Beyle.

$\mathrm{AN}, \mathrm{O}_{2}$ Io92, $\mathrm{n}^{\circ}$ 9, 74, 75 (le $\mathrm{n}^{\circ} 74$ est attaché par une épingle au n ${ }^{\circ} 75$ ), 73. 


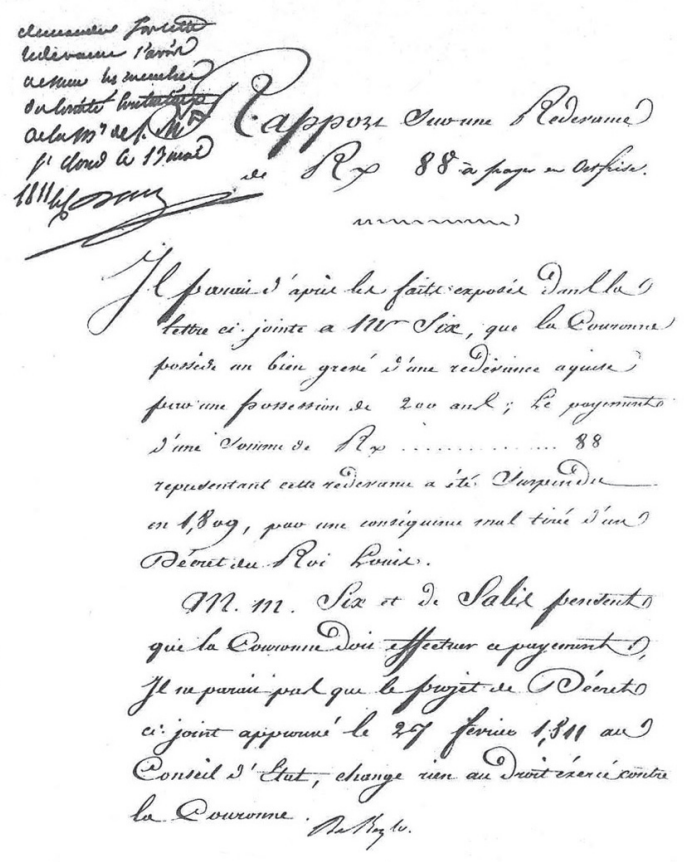

104

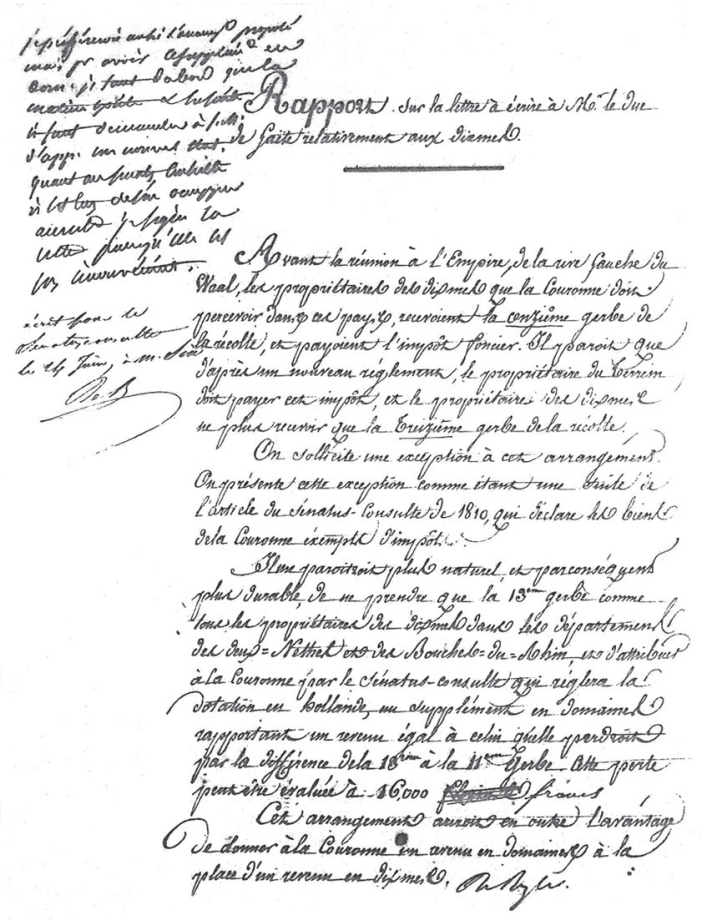

gt

Fig. 5. Rapports de Beyle, signatures et ajouts autographes; apostilles marginales autographes de Daru et de Beyle, mai et juin I8II.

$\mathrm{AN}, \mathrm{O}_{2}$ I092, $\mathrm{n}^{\circ}$ IO4, 97. 

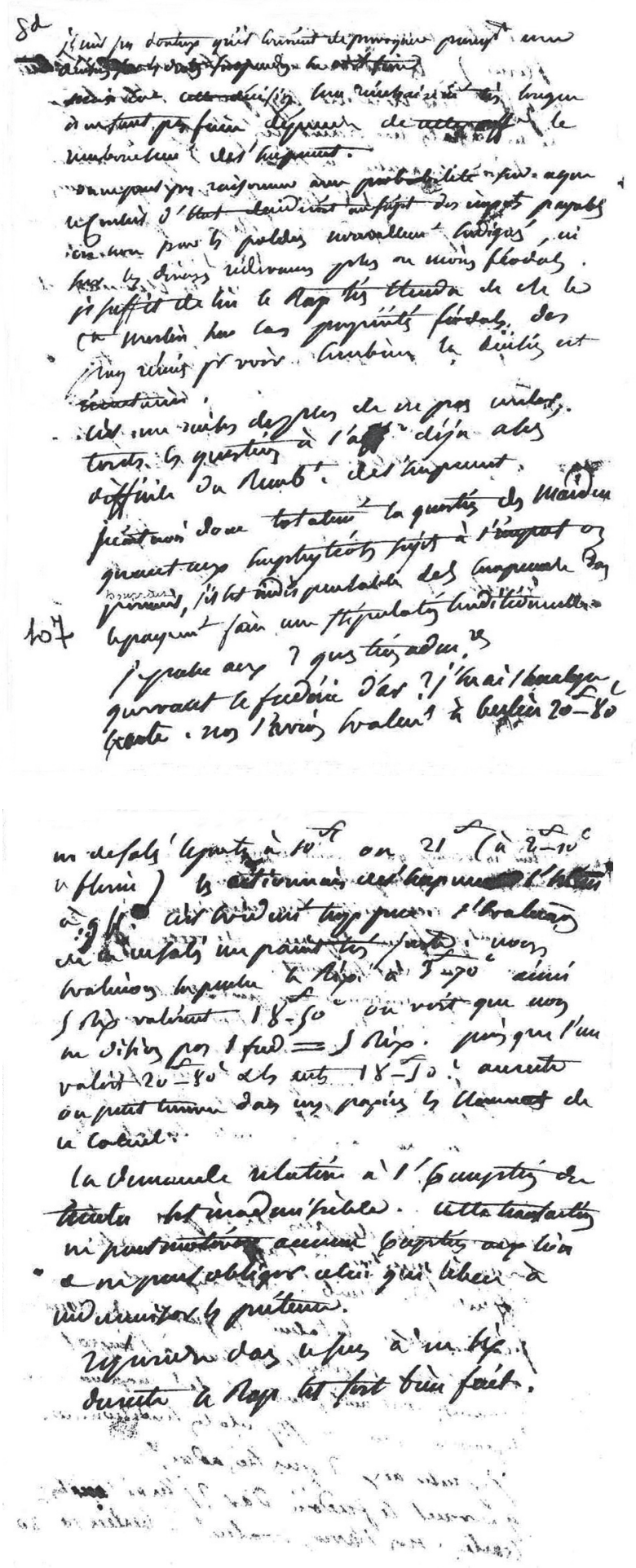

Fig. 6. Note autographe de Daru à Beyle, s. d. [2 avril I8II]. AN, O2 I092, nº 107. 


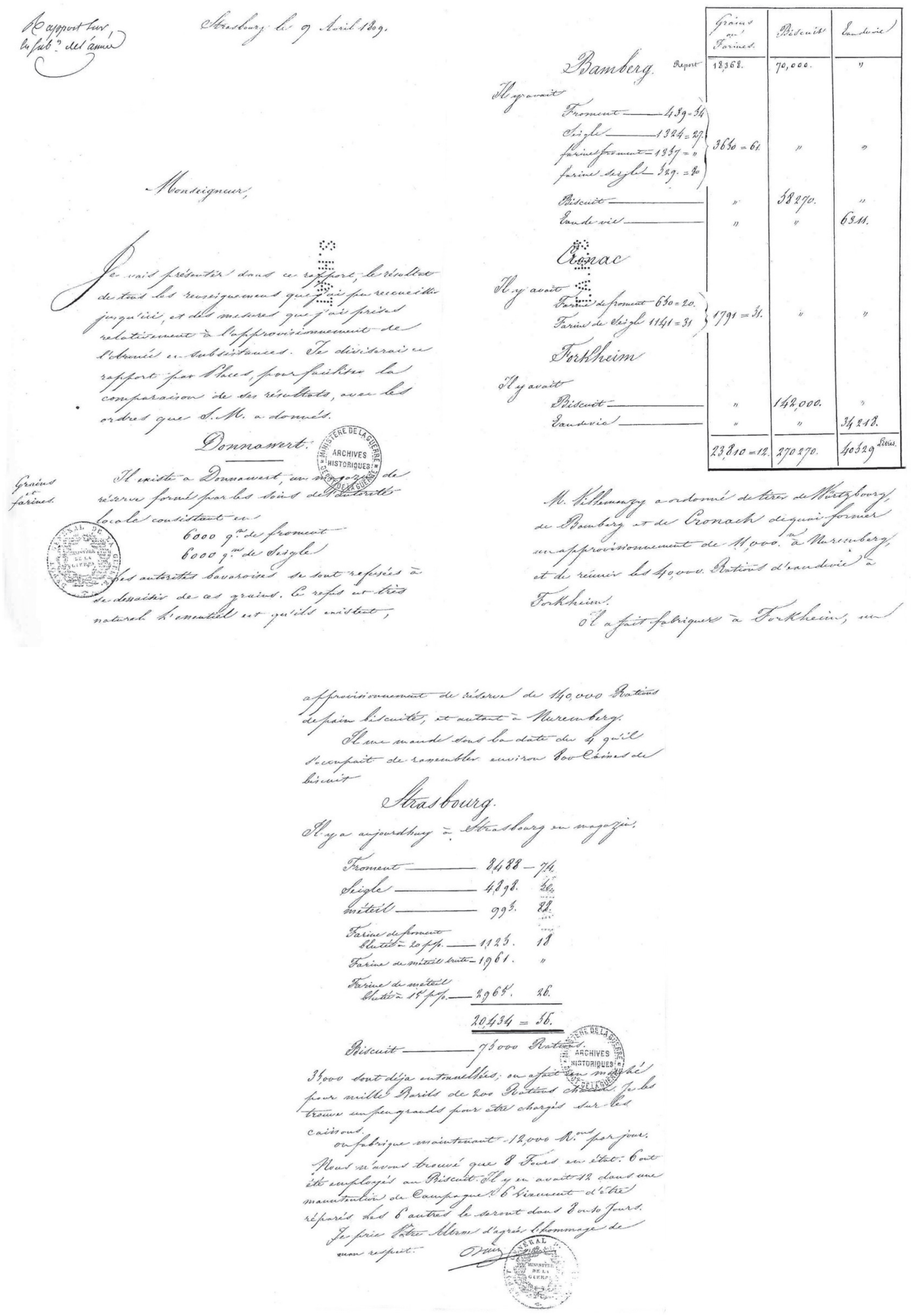

Fig. 7. Rapport de Daru à Berthier sur les subsistances de l'armée, de la main de Beyle, signature de Daru, 9 avril I809.

ASHAT, 2 C 87, p. I, I6, 17. 


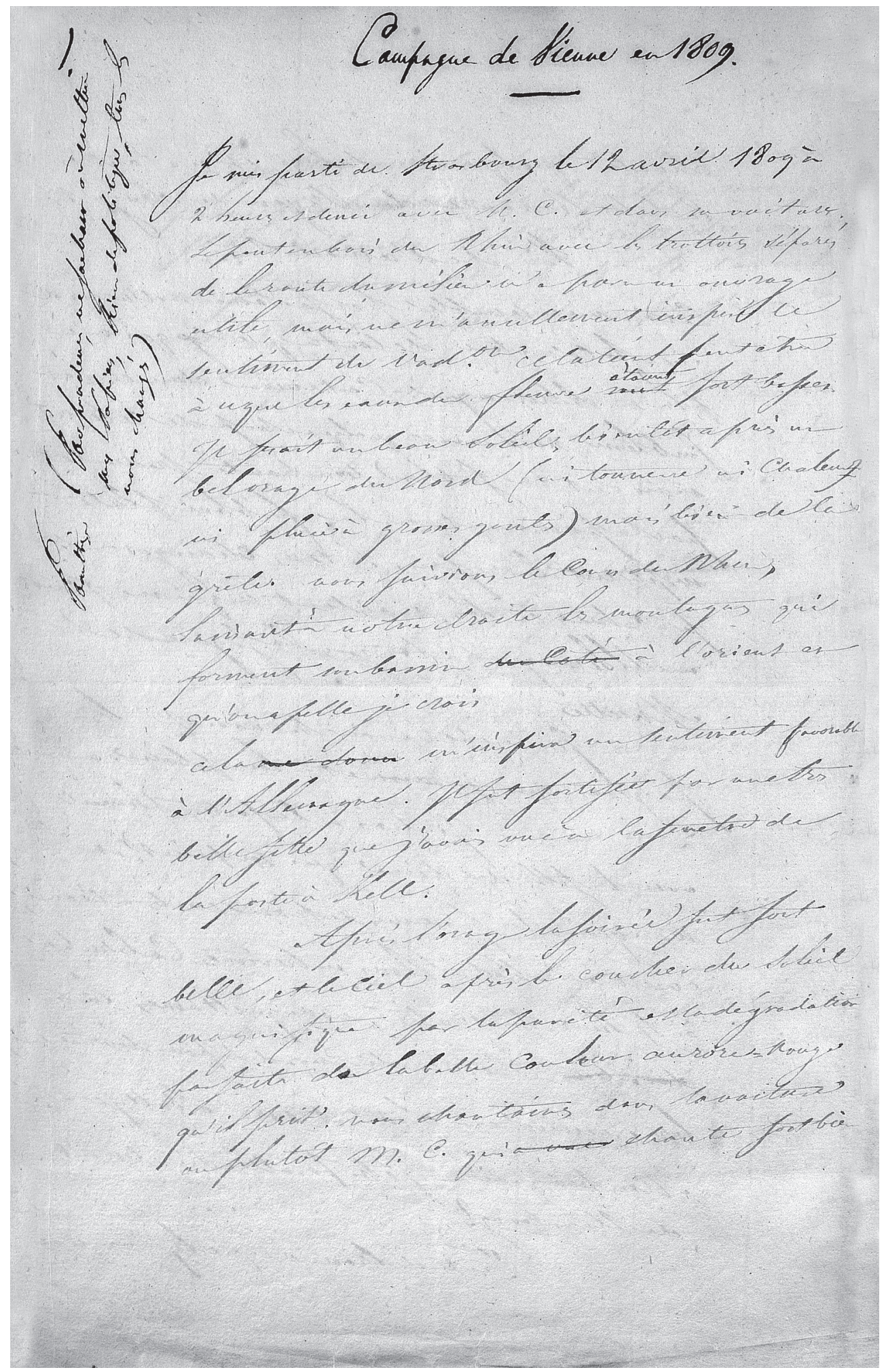

Fig. 8. Beyle, Journal, «Campagne de Vienne en I809", manuscrit autographe. BMG, R. 5896, vol. 5 , f II6v'. 


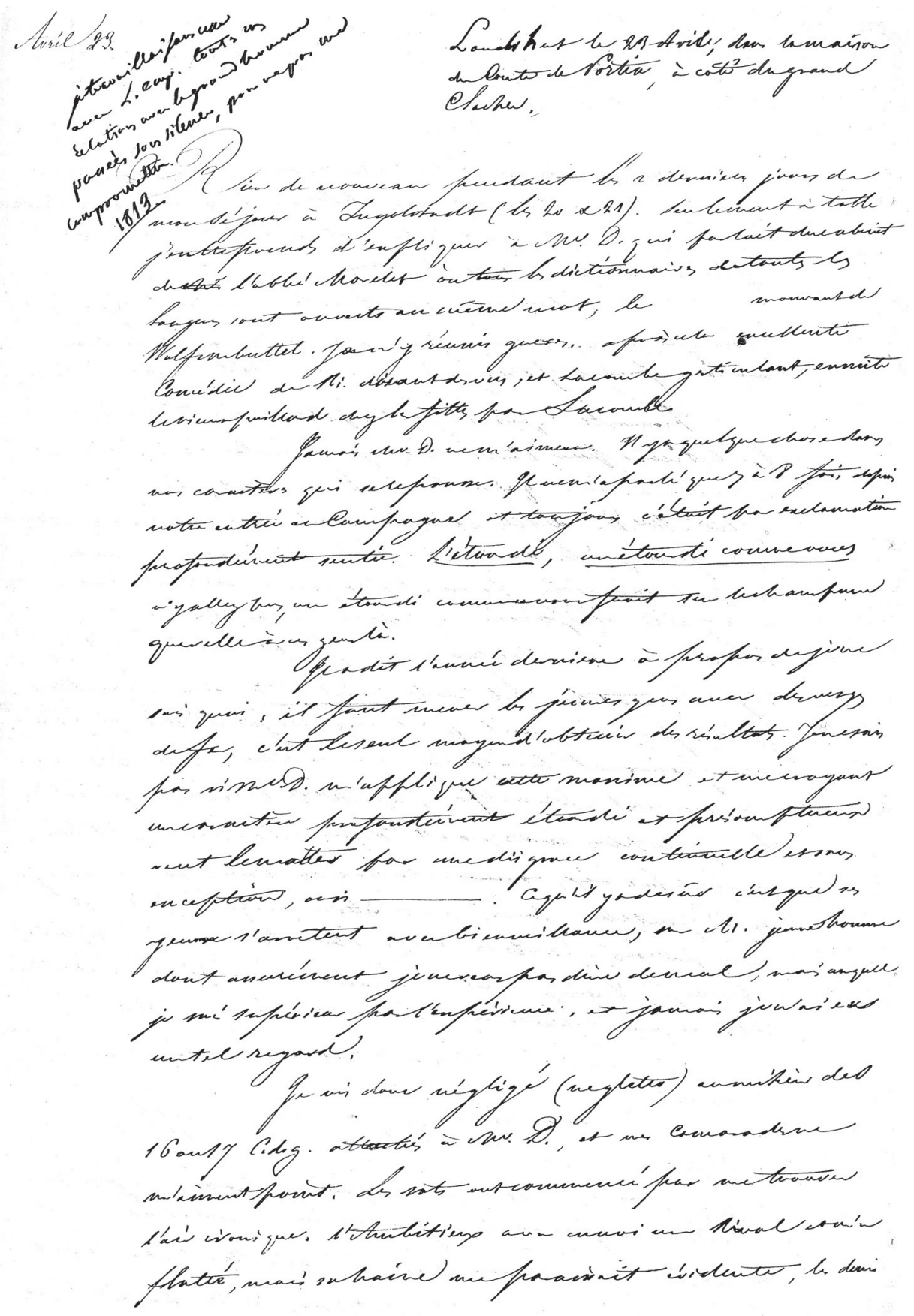

Fig. 9. Beyle, Journal, "Campagne de Vienne en I809", manuscrit autographe. BMG, R. 5896, vol. 5, f 109v'. 


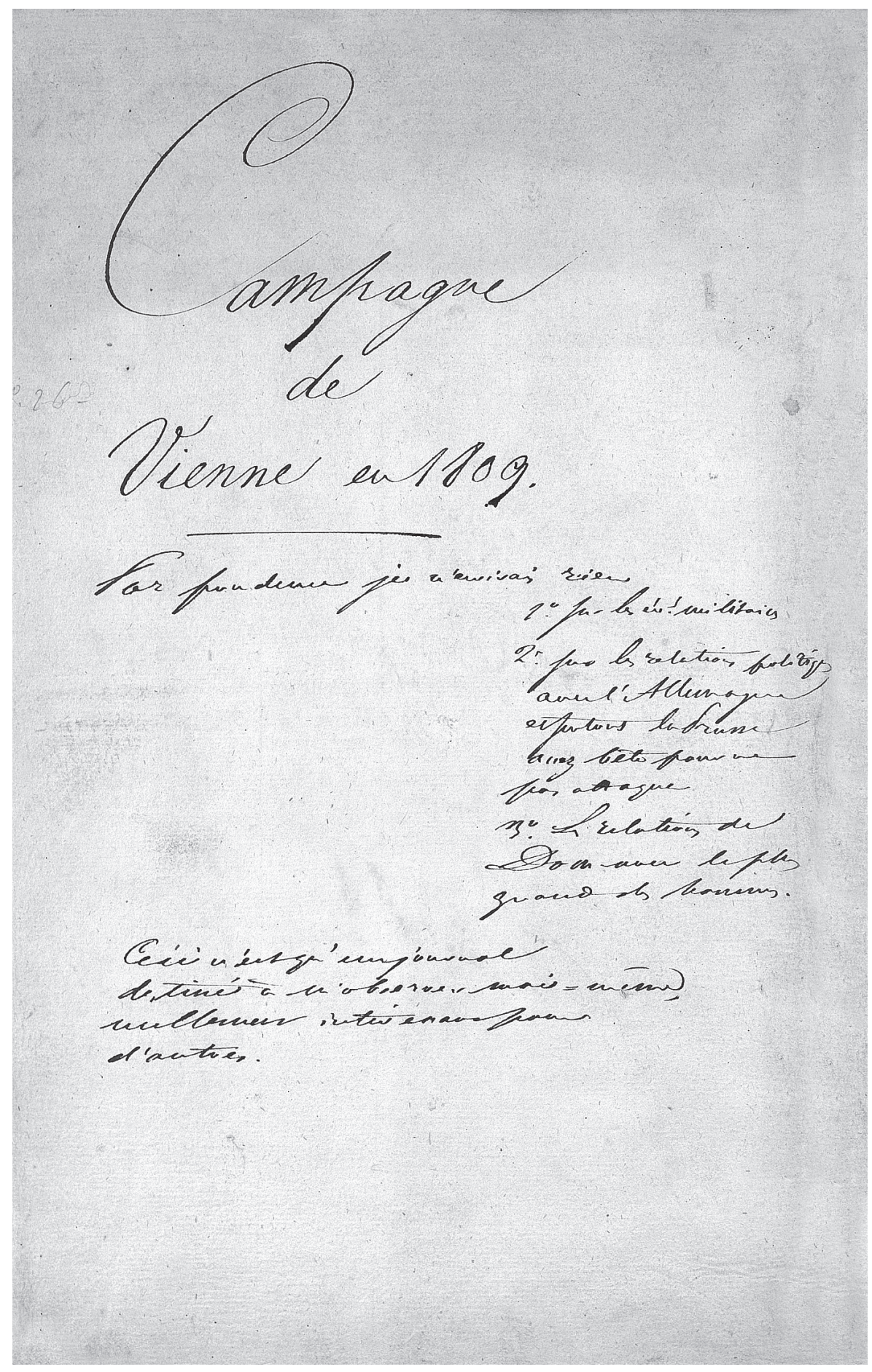

Fig. Io. Beyle, Journal, «Campagne de Vienne en I809", manuscrit autographe. BMG, R. 5896, vol. 5, f II $^{\circ} \mathrm{v}^{\circ}$. 


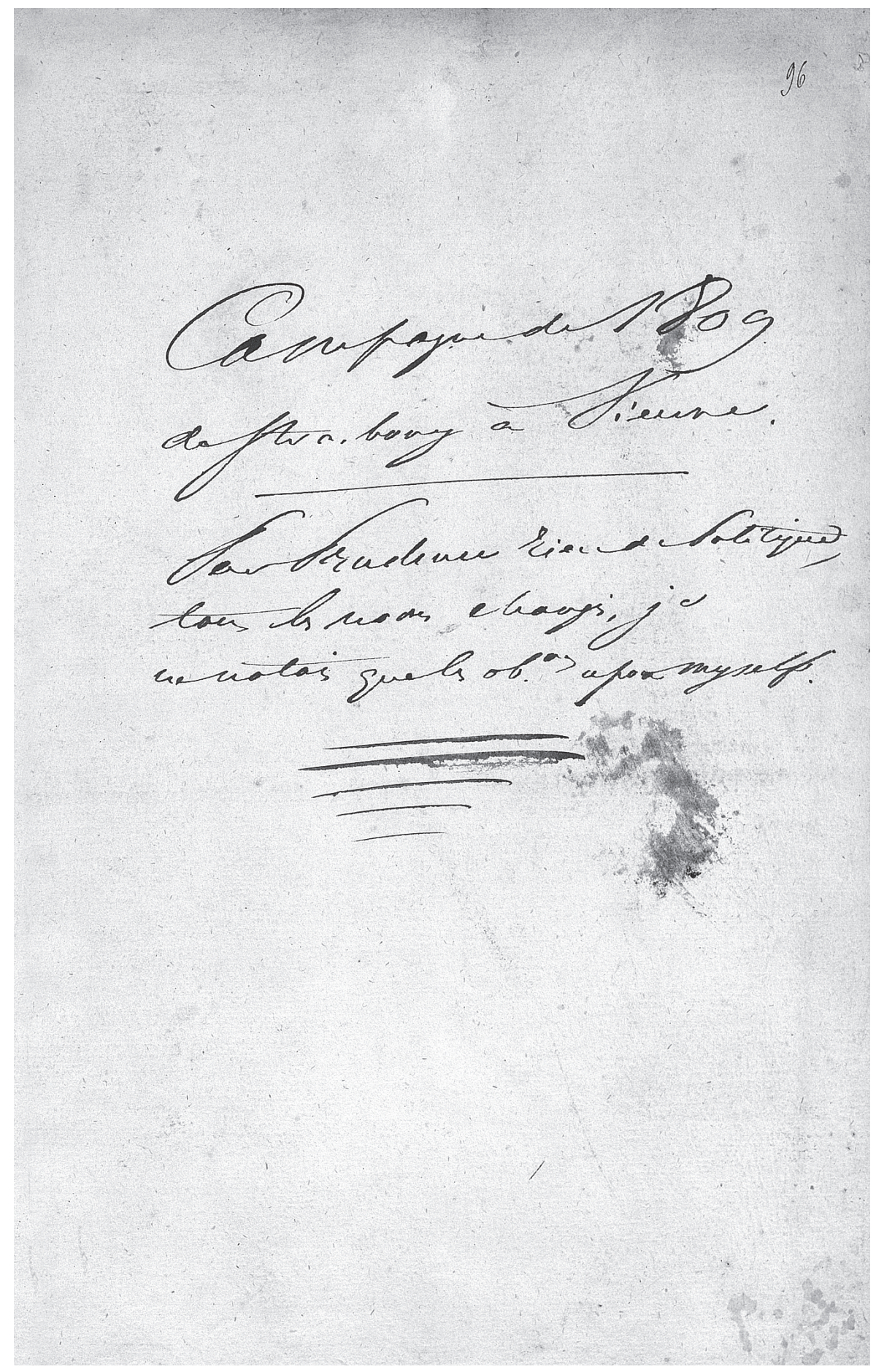

Fig. Ir. Beyle, Journal, «Campagne de Vienne en I809 », manuscrit autographe. BMG, R. 5896, vol. 5 , f $96 \mathrm{r}^{\circ}$ 


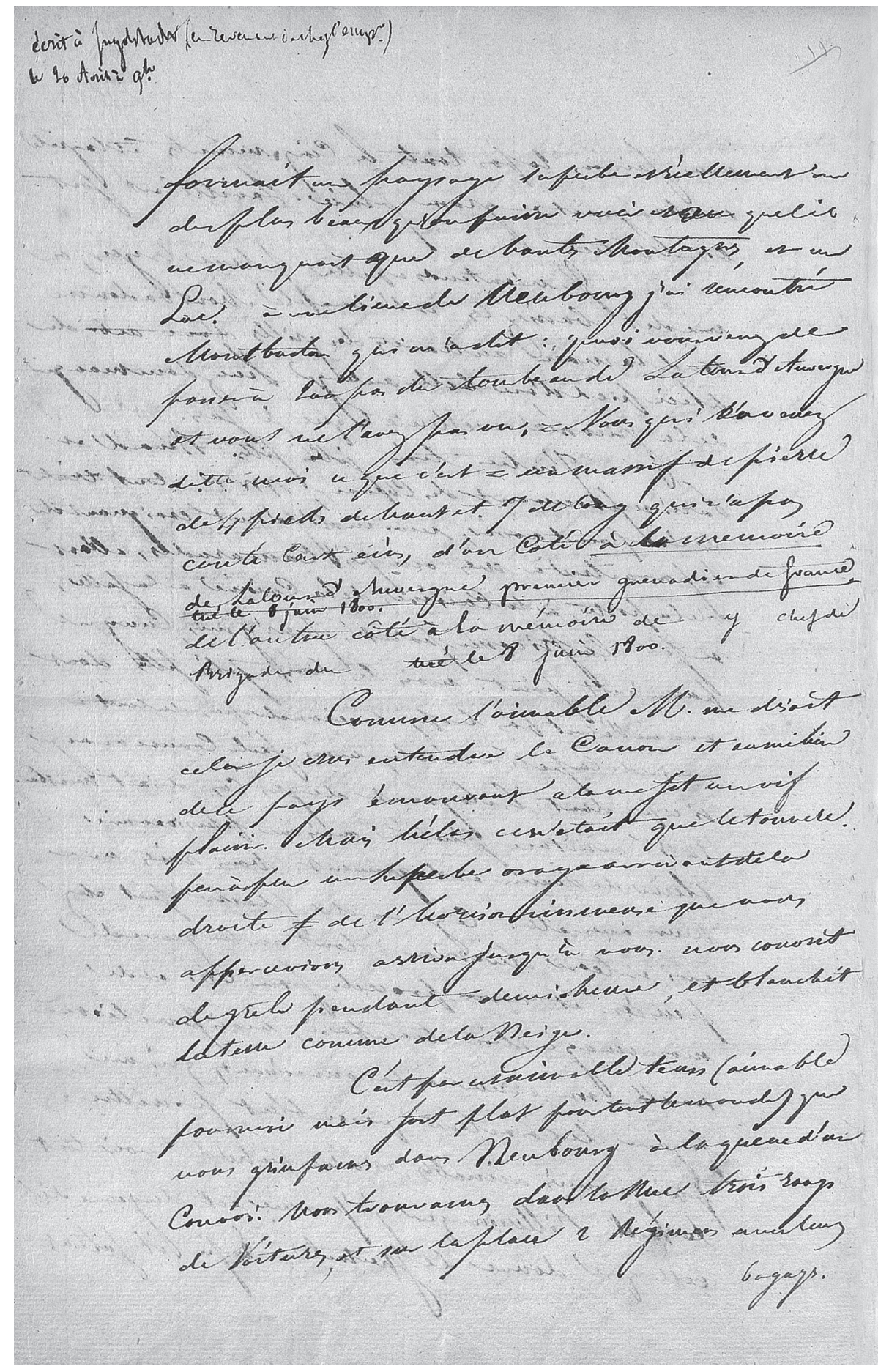

Fig. I2. Beyle, Journal, «Campagne de Vienne en I809 », manuscrit autographe. BMG, R. 5896, vol. $5, f^{\circ}$ IIIV $^{\circ}$. 

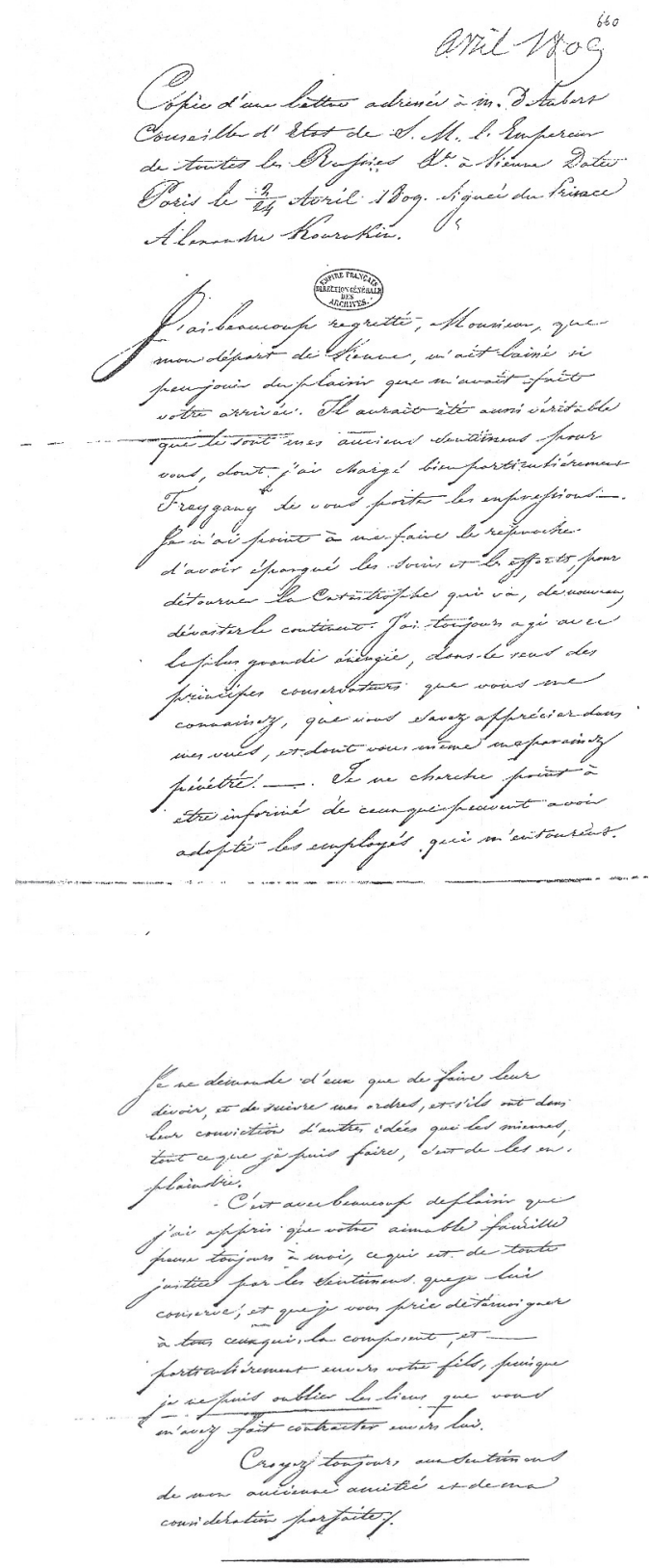

Fig. I3. Lettre du prince Kourakin à Aubert, copie autographe de Beyle adressée par Daru à Napoléon le 3 juin 1809 . AN, AF IV I677, pl. 2 III n ${ }^{\circ} 77, f^{\circ} 660$. 


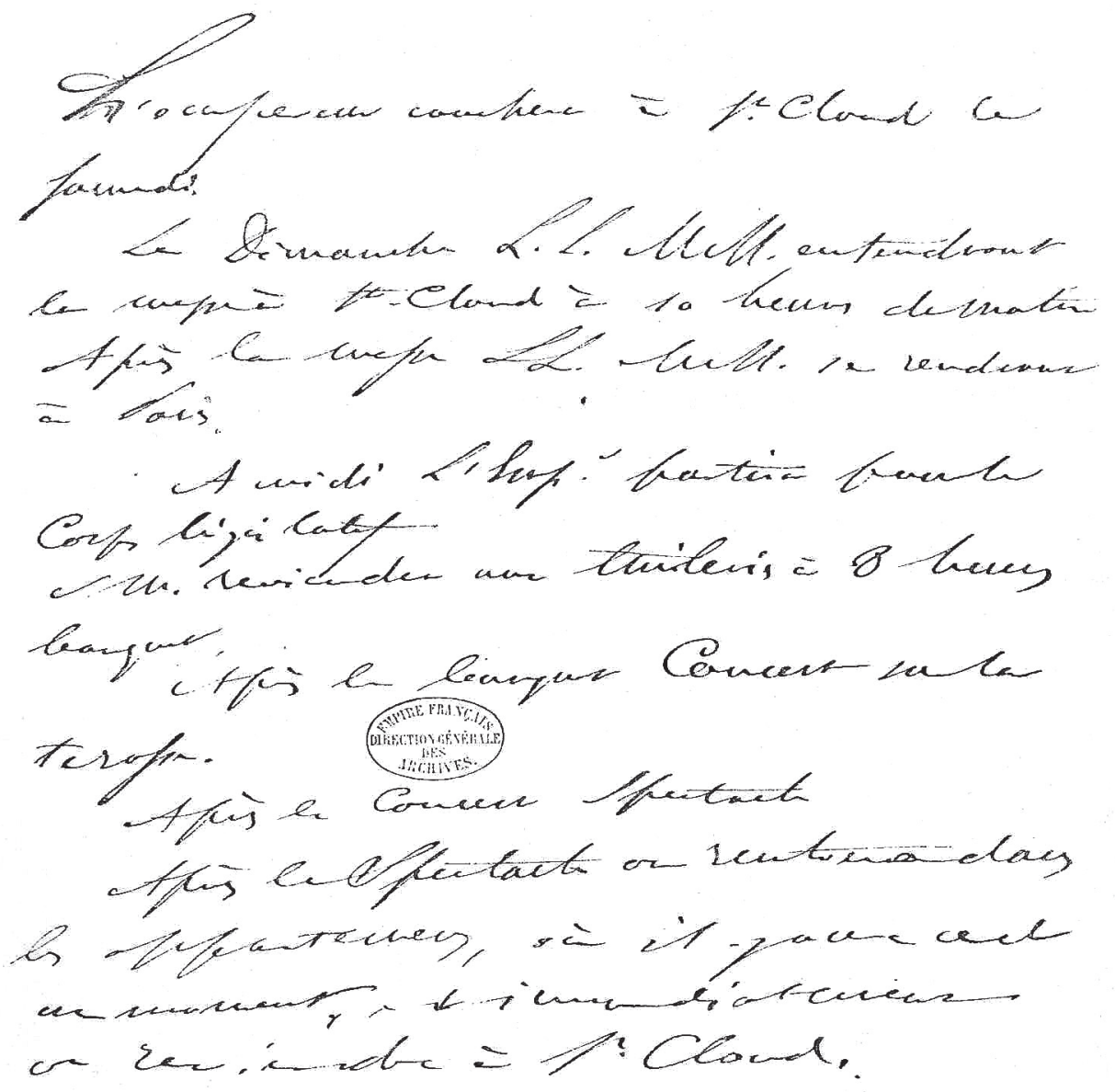

Fig. I4. Note autographe de Beyle, s. d. [vraisemblablement début mars I8Io]. $\mathrm{AN}, \mathrm{O}_{2} \mathrm{I} 56, \mathrm{n}^{\circ} 62$ 